\title{
ABSTRACT, CLASSIC, AND EXPLICIT TURNPIKES
}

\author{
PAOLO GUASONI, CONSTANTINOS KARDARAS, SCOTT ROBERTSON, AND HAO XING
}

\begin{abstract}
Portfolio turnpikes state that, as the investment horizon increases, optimal portfolios for generic utilities converge to those of isoelastic utilities. This paper proves three kinds of turnpikes. In a general semimartingale setting, the abstract turnpike states that optimal final payoffs and portfolios converge under their myopic probabilities. In diffusion models with several assets and a single state variable, the classic turnpike demonstrates that optimal portfolios converge under the physical probability; meanwhile the explicit turnpike identifies the limit of finite-horizon optimal portfolios as a long-run myopic portfolio defined in terms of the solution of an ergodic HJB equation.
\end{abstract}

\section{INTRODUCTION}

In the theory of portfolio choice, ruled by particular and complicated results, turnpike theorems are happy exceptions - general and simple. Loosely defined, these theorems state that, when the investment horizon is distant, the optimal portfolio of any investor approaches that of an investor with isoelastic utility, suggesting that for long-term investments, only isoelastic utilities matter.

This paper proves turnpike theorems in a general framework, which include discrete and continuous time, and nest diffusion models with several assets, stochastic drifts, volatilities, and interest rates. The paper departs from the existing literature, in which either asset returns are independent over time, or markets are complete. It is precisely when both these assumptions fail that portfolio choice becomes most challenging - and turnpike theorems are most useful.

Our results have three broad implications. First, turnpike theorems are a powerful tool in portfolio choice, because they apply not only when optimal portfolios are myopic, but also when the intertemporal hedging component is present. Finding this component is the central problem of portfolio choice, and the only tractable but non trivial analysis is based on isoelastic utilities, combined with long horizon asymptotics. Turnpike theorems make this analysis relevant for a large class of utility functions, and for large but finite horizons.

Second, we clarify the roles of preferences and market structure for turnpike results. Under regularity conditions on utility functions, we show that an abstract turnpike theorem holds regardless of

\footnotetext{
Key words and phrases. Portfolio Choice, Incomplete Markets, Long-Run, Utility Functions, Turnpikes.

Paolo Guasoni is partially supported by the ERC (278295), NSF (DMS-0807994, DMS-1109047), SFI (07/MI/008, 07/SK/M1189, 08/SRC/FMC1389), and FP7 (RG-248896).

Constantinos Kardaras is partially supported by the NSF DMS-0908461.
} 
market structure, as long as utility maximization is well posed, and longer horizons lead to higher payoffs. This abstract turnpike yields the convergence of optimal portfolios to their isoelastic limit under the myopic probability $\mathbb{P}^{T}$, which changes with the horizon. Market structure becomes crucial to pass from from the abstract to the classic turnpike theorem, in which convergence holds under the physical probability $\mathbb{P}$.

Third, in addition to the classical version, we prove a new kind of result, the explicit turnpike, in which the limit portfolio is identified as the long-run optimal portfolio, that is the solution to an ergodic Hamilton Jacobi Bellman equation. This result offers the first theoretical basis to the long-standing practice of interpreting solutions of ergodic HJB equations as long-run limits of utility maximization problems. We show that this intuition is indeed correct for a large class of diffusion models, and that its scope includes a broader class of utility functions.

Portfolio turnpikes start with the work of Mossin (1968) on affine risk tolerance $\left(-U^{\prime}(x) / U^{\prime \prime}(x)=\right.$ $a x+b)$, which envisions many of the later developments. In his concluding remarks, he writes: "Do any of these results carry over to arbitrary utility functions? They seem reasonable enough, but the generalization does not appear easy to make. As one usually characterizes those problems one hasn't been able to solve oneself: this is a promising area for future research".

Leland (1972) coins the expression portfolio turnpike, extending Mossin's result to larger classes of utilities, followed by Ross (1974) and Hakansson (1974). Huberman and Ross (1983) prove a necessary and sufficient condition for the turnpike property. As in the previous literature, they consider discrete time models with independent returns. Cox and Huang (1992) prove the first turnpike theorem in continuous time, using contingent claim methods. Jin (1998) extends their results to include consumption, and Huang and Zariphopoulou (1999) obtain similar results using viscosity solutions. Dybvig. Rogers, and Back (1999) dispose of the assumption of independent returns, proving a turnpike theorem for complete markets in the Brownian filtration, while Detemple and Rindisbacher (2010) obtain a portfolio decomposition formula for complete markets, which allows to compute turnpike portfolios in certain models.

In summary, the literature either exploits independent returns, which make dynamic programming attractive, or complete markets, which make martingale methods convenient. Since market completeness and independence of returns have a tenuous relation, neither of these concepts appears to be central to turnpike theorems. We confirm this intuition, by relaxing both assumptions in this paper.

The main results are in section 2, which is divided into two parts. The first part shows the conditions leading to the abstract turnpike, whereby optimal final payoffs and portfolios converge

\footnotetext{
${ }^{1}$ This interpretation underpins the literature on risk-sensitive control, introduced by Fleming and McEneaney (1995), and applied to optimal portfolio choice by Fleming and Sheu (2000; 2002), Bielecki et al. (2000; 2002), Nagai and Peng (2002b; 2002a) among others.
} 
under the myopic probabilities $\mathbb{P}^{T}$. Regarding preferences (Assumption 2.1), we require a marginal utility that is asymptotically isoelastic as wealth increases (CONV), and a well-posed utility maximization problem.

The second part of section 2 states the classic and explicit turnpike theorems for a class of diffusion models with several assets, but with a single state variable driving expected returns, volatilities and interest rates. Under further well-posedness assumptions, we show a classic turnpike theorem, in which optimal portfolios of generic utility functions converge to their isoelastic counterparts. The same machinery leads to the explicit turnpike, in which optimal portfolios for a generic utility and a finite horizon converge to the long-run optimal portfolio, that is the solution of an ergodic HJB equation. We conclude section 2 with an application to target-date retirement funds, which shows that a fund manager who tries to maximize the weighted welfare of participants - like a social planner - tends to act on behalf of the least risk averse investors.

Section 3 contains the proofs of the abstract turnpike, while the classic and the explicit turnpike for diffusions are proved in section 4 . The first part of section 3 proves the convergence of the ratio of final payoffs, while the second part derives the convergence of wealth processes. Section 4 studies the properties of the long-run measure and the value function, and continues with the convergence of densities and wealth processes, from which the classic and explicit turnpikes follow.

In conclusion, this paper shows that turnpike theorems are an useful tool to make portfolio choice tractable, even in the most intractable setting of incomplete markets combined with stochastic investment opportunities. Still, these results are likely to admit extensions to more general settings, like diffusions with multiple state variables, and processes with jumps. As gracefully put by Mossin, this is a promising area for future research.

\section{MAIN RESUlts}

This section contains the statements of the main results and their implications. The first subsection states an abstract version of the turnpike theorem, which focuses on payoff spaces and wealth processes, without explicit reference to the structure of the underlying market. In this setting, asymptotic conditions on the utility functions and on wealth growth imply that, as the horizon increases, optimal wealths and optimal portfolios converge to their isoelastic counterparts.

The defining feature of the abstract turnpike is that convergence takes place under a family of myopic probability measures that change with the horizon. By contrast, in the classic turnpike the convergence holds under the physical probability measure. Thus, passing from the abstract to the classic turnpike theorem requires the convergence of the myopic probabilities, which in turn commands additional assumptions. The second subsection achieves this task for a class of diffusion models with several risky assets, and with a single state variable driving investment opportunities. This class nests several models in the literature, and allows for return predictability, stochastic volatility, and stochastic interest rates. 
The explicit turnpike - stated at the end of the second subsection - holds for the same class of diffusion models. While in the abstract and classical turnpikes the benchmark is the optimal portfolio for isoelastic utility, but with the same finite horizon, in the explicit turnpike the benchmark is the long-run optimal portfolio, that is the optimal portfolio for asymptotic expected utility.

2.1. The Abstract Turnpike. Consider two investors, one with Constant Relative Risk Aversion (henceforth CRRA) equal to $1-p$ (i.e. power utility $x^{p} / p$ for $0 \neq p<1$ or logarithmic utility $\log x$ for $p=0$ ), the other with a generic utility function $U: \mathbb{R}_{+} \rightarrow \mathbb{R}$. The marginal utility ratio $\Re(x)$ measures how close $U$ is to the reference utility:

$$
\mathfrak{R}(x):=\frac{U^{\prime}(x)}{x^{p-1}}, \quad x>0 .
$$

Assumption 2.1. The utility function $U: \mathbb{R}_{+} \rightarrow \mathbb{R}$ is continuously differentiable, strictly increasing, strictly concave, and satisfies the Inada conditions $U^{\prime}(0)=\infty$ and $U^{\prime}(\infty)=0$. The marginal utility ratio satisfies:

$$
\lim _{x \uparrow \infty} \mathfrak{R}(x)=1 .
$$

Condition (CONV) means that investors have similar marginal utilities when wealth is high, and is the basic assumption on preferences for turnpike theorems (Dybvig et al., 1999; Huang and Zariphopoulou, 1999).

Both investors trade in a frictionless market with one safe and $d$ risky assets. Consider a filtered probability space $\left(\Omega,\left(\mathcal{F}_{t}\right)_{t \in[0, T]}, \mathcal{F}, \mathbb{P}\right)$, where $\left(\mathcal{F}_{t}\right)_{t \geq 0}$ is a right-continuous filtration. The safe asset, denoted by $\left(S_{t}^{0}\right)_{t \geq 0}$ and the risky assets $\left(S_{t}^{i}\right)_{t \geq 0}^{1 \leq i \leq d}$ satisfy the following:

Assumption 2.2. $S^{0}$ has RCLL (right-continuous, left-limited) paths, and there exist two deterministic functions $\underline{S}^{0}, \bar{S}^{0}: \mathbb{R}_{+} \mapsto \mathbb{R}_{+}$, such that $0<\underline{S}_{t}^{0} \leq S_{t}^{0} \leq \bar{S}_{t}^{0}$ for all $t>0$ and

$$
\lim _{T \rightarrow \infty} \underline{S}_{T}^{0}=\infty
$$

This condition means that growth continues over time, and is the main market assumption in the turnpike literature. It implies, that the riskless discount factor declines to zero in the long run. Now, denote the discounted prices of risky assets by $\widetilde{S}^{i}=S^{i} / S^{0}$ for $i=1, \ldots, d$, and set $\widetilde{S}=\left(\widetilde{S}_{t}^{1}\right)_{t \geq 0}^{1 \leq i \leq d}$. The following assumption is equivalent to the absence of arbitrage, in the sense of No Free Lunch with Vanishing Risk (Delbaen and Schachermaver, 1994, 1998). In particular, up to a null set, $S=\left(S_{t}^{i}\right)_{t \geq 0}^{1 \leq i \leq d}$ is a $\mathbb{R}^{d}$-valued semimartingale with RCLL paths.

Assumption 2.3. For all $T \in \mathbb{R}_{+}$, there exists a probability $\mathbb{Q}^{T}$ that is equivalent to $\mathbb{P}$ on $\mathcal{F}_{T}$ and such that $\widetilde{S}$ is a (vector) sigma-martingale.

Starting from unit capital, each investor trades with some admissible strategy $H$, that is a $S$-integrable and $\mathcal{F}$-predictable $\mathbb{R}^{d}$-valued process, such that $\widetilde{X}_{t}^{H}:=1+\int_{0}^{t} H_{u} d \widetilde{S}_{u} \geq 0 \mathbb{P}$-a.s. 
for all $t \geq 0$. Denote a wealth process by $X^{H}=S^{0} \widetilde{X}^{H}$, and their class by $\mathcal{X}^{T}:=\left\{X^{H}\right.$ : $H$ is $T$-admissible $\}$.

Both investors seek to maximize the expected utility of their terminal wealth at some time horizon $T$. Using the index 0 for the CRRA investor, and 1 for the generic investor, their optimization problems are:

$$
u^{0, T}=\sup _{X \in \mathcal{X}^{T}} \mathbb{E}^{\mathbb{P}}\left[X_{T}^{p} / p\right], \quad u^{1, T}=\sup _{X \in \mathcal{X}^{T}} \mathbb{E}^{\mathbb{P}}\left[U\left(X_{T}\right)\right] .
$$

The next assumption requires that these problems are well-posed. It holds under the simple criteria in Karatzas and Žitković (2003, Remark 8).

Assumption 2.4. For all $T>0$ and $i=0,1, u^{i, T}<\infty$.

Karatzas and Žitković (2003) show that, under Assumptions 2.1 2.4, the optimal wealth processes $X^{i, T}$ exist for $i=0,1$ and any $T \geq 0$. In addition, $u^{i, T}>-\infty$, because both investors can invest all their wealth in $S^{0}$ alone, and $S_{T}^{0}$ is bounded away from zero by a constant.

The central objects in the abstract turnpike theorem are the ratio of optimal wealth processes and their stochastic logarithms

$$
r_{u}^{T}:=\frac{X_{u}^{1, T}}{X_{u}^{0, T}}, \quad \Pi_{u}^{T}:=\int_{0}^{u} \frac{d r_{v}^{T}}{r_{v-}^{T}}, \quad \text { for } u \in[0, T],
$$

and are well-defined by Remark 3.2 below. Moreover, $r_{0}^{T}=1$ since both investors have the same initial capital. Define also the myopic probabilities $\left(\mathbb{P}^{T}\right)_{T \geq 0}$ by:

$$
\frac{d \mathbb{P}^{T}}{d \mathbb{P}}=\frac{\left(X_{T}^{0, T}\right)^{p}}{\mathbb{E}^{\mathbb{P}}\left[\left(X_{T}^{0, T}\right)^{p}\right]} .
$$

The above densities are well-defined and strictly positive (cf. Assumption 2.4 and Remark 3.2), and $\mathbb{P}^{T}=\mathbb{P}$ in the logarithmic case $p=0$. These myopic probabilities are interpreted as follows: an investor with relative risk aversion $1-p$ under the probability $\mathbb{P}$ selects the same optimal payoff as another investor under the probability $\mathbb{P}^{T}$, but with logarithmic utility, that is with unit risk aversion 2 .

With the above definitions, the abstract version of the turnpike theorem reads as follows:

Theorem 2.5 (Abstract Turnpike). Let Assumptions 2.1 2.4 hold. Then, for any $\epsilon>0$,

a) $\lim _{T \rightarrow \infty} \mathbb{P}^{T}\left(\sup _{u \in[0, T]}\left|r_{u}^{T}-1\right| \geq \epsilon\right)=0$,

b) $\lim _{T \rightarrow \infty} \mathbb{P}^{T}\left(\left[\Pi^{T}, \Pi^{T}\right]_{T} \geq \epsilon\right)=0$, where $[\cdot, \cdot]$ denotes the square bracket of semimartingales.

Remark 2.6.

\footnotetext{
${ }^{2}$ These probabilities already appear in the work of Kramkov and Sîrbu (2006a , b, 2007) under the name of R.
} 
i) Since $\mathbb{P}^{T} \equiv \mathbb{P}$ for $p=0$, convergence holds under $\mathbb{P}$ in the case of logarithmic utility. In particular, the convergence holds on the entire time horizon $[0, T]$. Contrast this to the turnpike results for $p \neq 0$, in which convergence holds on a time window $[0, t]$ for some fixed $t>0$.

ii) Consider a market with the discounted asset prices

$$
\frac{d \widetilde{S}_{u}^{j}}{\widetilde{S}_{u}^{j}}=\mu_{u}^{j} d u+\sum_{k=1}^{n} \sigma_{u}^{j k} d W_{u}^{k}, \quad j=1, \cdots, d,
$$

where $\mu_{u}=\in \mathbb{R}^{d}, \sigma_{u} \in \mathbb{R}^{d \times n}$ for $t \geq 0$ and $W=\left(W^{1}, \cdots, W^{n}\right)^{\prime}$ is a $\mathbb{R}^{n}$-valued Brownian motion. The discounted optimal wealth processes satisfy

$$
d \widetilde{X}_{u}^{i, T}=\widetilde{X}_{u}^{i, T}\left(\pi_{u}^{i, T}\right)^{\prime}\left(\mu_{u} d u+\sigma_{u} d W_{u}\right), \quad i=0,1,
$$

where $\left(\pi^{i, T}\right)_{u \geq 0}^{1 \leq j \leq d}$ represents the proportions of wealth invested in each risky asset. In this case, $\left[\Pi^{T}, \Pi^{T}\right]$ measures the square distance, weighted by $\Sigma=\sigma \sigma^{\prime}$, between the portfolios $\pi^{1, T}$ and $\pi^{0, T}$ :

$$
\left[\Pi^{T}, \Pi^{T}\right] .=\int_{0}^{\cdot}\left(\pi_{u}^{1, T}-\pi_{u}^{0, T}\right)^{\prime} \Sigma_{u}\left(\pi_{u}^{1, T}-\pi_{u}^{0, T}\right) d u .
$$

iii) The theorem implies that both optimal wealth processes and portfolios are close in any time window $[0, t]$ for any fixed $t>0$, under the probability $\mathbb{P}^{T}$. Indeed, for any $\epsilon, t>0$ :

$$
\lim _{T \rightarrow \infty} \mathbb{P}^{T}\left(\sup _{u \in[0, t]}\left|r_{u}^{T}-1\right| \geq \epsilon\right)=0 \quad \text { and } \quad \lim _{T \rightarrow \infty} \mathbb{P}^{T}\left(\left[\Pi^{T}, \Pi^{T}\right]_{t} \geq \epsilon\right)=0 .
$$

Except for logarithmic utility, Theorem 2.5 is not a classic turnpike theorem, in that convergence holds under the probabilities $\mathbb{P}^{T}$, which change with the horizon $T$. However, since the events $\left\{\sup _{u \in[0, t]}\left|r_{u}^{T}-1\right| \geq \epsilon\right\}$ and $\left\{\left[\Pi^{T}, \Pi^{T}\right]_{t} \geq \epsilon\right\}$ are $\mathcal{F}_{t}$-measurable, and any such event $A$ satisfies $\mathbb{P}^{T}(A)=\mathbb{E}^{\mathbb{P}}\left[1_{A} d \mathbb{P}^{T} /\left.d \mathbb{P}\right|_{\mathcal{F}_{t}}\right]$, the relation between $\mathbb{P}^{T}(A)$ and $\mathbb{P}(A)$ depends on the (projected) density:

$$
\left.\frac{d \mathbb{P}^{T}}{d \mathbb{P}}\right|_{\mathcal{F}_{t}}=\frac{\mathbb{E}_{t}^{\mathbb{P}}\left[\left(X_{T}^{0, T}\right)^{p}\right]}{\mathbb{E}^{\mathbb{P}}\left[\left(X_{T}^{0, T}\right)^{p}\right]}
$$

Understanding the convergence of these densities is the crucial step to bridge the gap from the abstract to the classic version of the turnpike theorem.

In fact, the densities in (2.5) become trivial under two additional assumptions: that the optimal CRRA strategy is myopic, and that its wealth process has independent returns. Under these assumptions, which hold in all the turnpike literature, with the exception of (Dybvig et al., 1999, Theorem 1), the density $d \mathbb{P}^{T} /\left.d \mathbb{P}\right|_{\mathcal{F}_{t}}$ is independent of $T$, and the classic turnpike theorem follows:

Corollary 2.7 (IID Myopic Turnpike). If, in addition to Assumptions 2.1 - 2.4.

(1) $X_{t}^{0, T}=X_{t}^{0, S} \equiv X_{t} \mathbb{P}$-a.s. for all $t \leq S, T$ (myopic optimality); 
(2) $X_{s} / X_{t}$ and $\mathcal{F}_{t}$ are independent under $\mathbb{P}$ for all $t \leq s$ (independent returns).

then, for any $\epsilon, t>0$ :

a) $\lim _{T \rightarrow \infty} \mathbb{P}\left(\sup _{u \in[0, t]}\left|r_{u}^{T}-1\right| \geq \epsilon\right)=0$,

b) $\lim _{T \rightarrow \infty} \mathbb{P}\left(\left[\Pi^{T}, \Pi^{T}\right]_{t} \geq \epsilon\right)=0$.

In practice, if asset prices have independent returns, the optimal strategy for a CRRA investor entails a myopic portfolio with independent returns, and both conditions above hold. This is the case, for example, if asset prices follow exponential Lévy processes, as in Kallsen (2000). Note however, that a myopic CRRA portfolio is not sufficient to ensure that $\mathbb{P}^{T}$ is independent of $T$ (cf. Example 2.22 below).

Thus, the abstract turnpike readily yields a classic turnpike theorem under additional assumptions in Corollary 2.7. However, even though these assumptions are common in the literature, they exclude models in which portfolio choice is least tractable, and turnpike results are needed the most. The next section proves classical and explicit turnpikes for diffusion models in which returns may not be independent, and the market may be incomplete.

2.2. A Turnpike for Diffusions. This subsection states the classic turnpike theorem for a class of diffusion models, in which a single state variable drives investment opportunities. The state variable takes values in some interval $E=(\alpha, \beta) \subseteq \mathbb{R}$, with $-\infty \leq \alpha<\beta \leq \infty$, and has the dynamics

$$
d Y_{t}=b\left(Y_{t}\right) d t+a\left(Y_{t}\right) d W_{t}
$$

The market includes a safe rate $r\left(Y_{t}\right)$ and $d$ risky assets, with prices $S_{t}^{i}$ satisfying

$$
\frac{d S_{t}^{i}}{S_{t}^{i}}=r\left(Y_{t}\right) d t+d R_{t}^{i}, \quad 1 \leq i \leq d,
$$

where the cumulative excess return process $R=\left(R^{1}, \cdots, R^{d}\right)^{\prime}$ is defined as

$$
d R_{t}^{i}=\mu_{i}\left(Y_{t}\right) d t+\sum_{j=1}^{d} \sigma_{i j}\left(Y_{t}\right) d Z_{t}^{j}, \quad 1 \leq i \leq d .
$$

Here $W$ and $Z=\left(Z^{1}, \cdots, Z^{d}\right)^{\prime}$ are Brownian motions with correlations $\rho=\left(\rho^{1}, \cdots, \rho^{d}\right)^{\prime}$, i.e. $d\left\langle Z^{i}, W\right\rangle_{t}=\rho^{i}\left(Y_{t}\right) d t$ for $1 \leq i \leq d$. (The prime sign is for matrix transposition.)

Denote by $\Sigma=\sigma \sigma^{\prime}, A=a^{2}$, and $\Upsilon=\sigma \rho a$. The first assumption on the model's coefficients concerns regularity and non-degeneracy. Recall that for $\gamma \in(0,1]$ and an integer $k$, a function $f: E \mapsto \mathbb{R}$ is locally $C^{k, \gamma}$ on $E$ if for all bounded, open, connected $D \subset E$ such that $\bar{D} \subset E$ it follows that $f \in C^{k, \gamma}(\bar{D})$ (see Evans (1998, Chapter 5.1) for a definition of the Hölder space $C^{k, \gamma}$ ). For integers $n, m, C^{k, \gamma}\left(E, \mathbb{R}^{n \times m}\right)$ is the set of all $n \times m$ matrix-valued $f$ for which each component $f_{i j}$ is locally $C^{k, \gamma}$ on $E$. Write $\mathbb{R}=\mathbb{R}^{1 \times 1}$ and $\mathbb{R}^{n}=\mathbb{R}^{n \times 1}$. With this notation, assume: 
Assumption 2.8. $r \in C^{\gamma}(E, \mathbb{R}), b \in C^{1, \gamma}(E, \mathbb{R}), \mu \in C^{1, \gamma}\left(E, \mathbb{R}^{d}\right), A \in C^{2, \gamma}(E, \mathbb{R}), \Sigma \in$ $C^{2, \gamma}\left(E, \mathbb{R}^{d \times d}\right)$, and $\Upsilon \in C^{2, \gamma}\left(E, \mathbb{R}^{d}\right)$ for some $\gamma \in(0,1]$. For all $y \in E, \Sigma$ is positive definite and $A$ is positive.

These regularity conditions imply the local existence and uniqueness of a solution to the joint dynamics of the state variable and asset prices. The next assumption ensures the existence of a unique global solution, by requiring that Feller's test for explosions is negative (Pinsky, 1995, Theorem 5.1.5).

Assumption 2.9. There is some $y_{0} \in E$ such that

$$
\int_{\alpha}^{y_{0}} \frac{1}{A(y) m(y)}\left(\int_{y}^{y_{0}} m(z) d z\right) d y=\infty=\int_{y_{0}}^{\beta} \frac{1}{A(y) m(y)}\left(\int_{y_{0}}^{y} m(z) d z\right) d y
$$

where the speed measure is defined as $m(y):=\frac{1}{A(y)} \exp \left(\int_{y_{0}}^{y} \frac{2 b(z)}{A(z)} d z\right)$.

Assumption 2.9 implies the model for $(R, Y)$ is well posed in that it admits a solution. This statement is made precise within the setting of the martingale problem, now introduced along with some notation. Let $\Omega$ be the space of continuous maps $\omega: \mathbb{R}_{+} \rightarrow \mathbb{R}^{n}$ and $\left(\mathcal{B}_{t}\right)_{t \geq 0}$ be the filtration generated by the coordinate process $\Xi$ defined by $\Xi_{t}(\omega)=\omega_{t}$ for $\omega \in \Omega$. Let $\mathcal{F}=\sigma\left(\Xi_{t}, t \geq 0\right)$ and $\mathcal{F}_{t}=\mathcal{B}_{t+}$. For an open, connected set $D \subset \mathbb{R}^{n}$ and $\gamma \in(0,1]$, let $\widetilde{A} \in C^{2, \gamma}\left(D, \mathbb{R}^{n \times n}\right)$ be point-wise positive definite and let $\widetilde{b} \in C^{1, \gamma}\left(D, \mathbb{R}^{n}\right)$. Define the second order elliptic operator $\widetilde{L}$ by

$$
\widetilde{L}=\frac{1}{2} \sum_{i, j=1}^{n} \widetilde{A}_{i j} \frac{\partial^{2}}{\partial x_{i} \partial x_{j}}+\sum_{i=1}^{n} \widetilde{b}_{i} \frac{\partial}{\partial x_{i}}
$$

Definition 2.10. A family of probability measures $\left(\mathbb{P}^{x}\right)_{x \in D}$ on $(\Omega, \mathcal{F})$ is a solution to the martingale problem for $\widetilde{L}$ on $D$ if, for each $\left.x \in D: i): \mathbb{P}^{x}\left(\Xi_{0}=x\right)=1, i i\right): \mathbb{P}^{x}\left(\Xi_{t} \in D, \forall t \geq 0\right)=1$, and iii $):\left(f\left(\Xi_{t}\right)-f\left(\Xi_{0}\right)-\int_{0}^{t} \widetilde{L} f\left(\Xi_{u}\right) d u ;\left(\mathcal{F}_{t}\right)_{t \geq 0}\right)$ is a $\mathbb{P}^{x}$ martingale for all $f \in C_{0}^{2}(D)$.

Let $\xi=(z, y) \in \mathbb{R}^{d} \times E$. Consider the generator

$$
L=\frac{1}{2} \sum_{i, j=1}^{d+1} \widetilde{A}_{i j}(\xi) \frac{\partial^{2}}{\partial \xi_{i} \partial \xi_{j}}+\sum_{i=1}^{d+1} \widetilde{b}_{i}(\xi) \frac{\partial}{\partial \xi_{i}}, \quad \widetilde{A}=\left(\begin{array}{cc}
1_{d} & 0 \\
0 & A
\end{array}\right) \text { and } \widetilde{b}=\left(\begin{array}{l}
0 \\
b
\end{array}\right) .
$$

This is the infinitesimal generator of $(B, Y)$ from (2.6) and (2.7) where $B$ is a $d$-dimensional Brownian Motion starting at $z$, independent of $Y$ which starts at $y$. Assumptions 2.8 and 2.9 imply the following:

Proposition 2.11. Let Assumptions 2.8 and 2.9 hold. Then there exists a unique solution $\left(\mathbb{P}^{\xi}\right)_{\xi \in \mathbb{R}^{d} \times E}$ to the martingale problem on $\mathbb{R}^{d} \times E$ for $L$ in (2.8).

Remark 2.12. There is a one to one correspondence between solutions to the martingale problem and weak solutions for $(B, Y)$, see (Rogers and Williams, 2000, Chapter V). Since $A(y)>0$ for 
$y \in E$, defining $W$ via $W_{t}=\int_{0}^{t} a\left(Y_{s}\right)^{-1}\left(d Y_{s}-b\left(Y_{s}\right) d s\right), Z=\rho W+\bar{\rho} B$ where $\bar{\rho}$ is a square root of $1-\rho \rho^{\prime}$, and $R$ via (2.7), it follows that $\left((R, Y),(W, B),\left(\Omega, \mathcal{F},\left(\mathcal{F}_{t}\right)_{t \geq 0}, \mathbb{P}^{\xi}\right)\right)$ is a weak solution of (2.6) and (2.7).

Assumption 2.9 is merely technical, in that it requires that the original market is well defined. By contrast, the next assumption places some restrictions on market dynamics.

Assumption 2.13. $\rho^{\prime} \rho$ is constant (i.e. it does not depend on $y$ ), and $\sup _{y \in E} c(y)<\infty$, where

$$
c(y):=\frac{1}{\delta}\left(p r-\frac{q}{2} \mu^{\prime} \Sigma^{-1} \mu\right)(y), \quad y \in E
$$

with $q:=p /(p-1)$ and $\delta:=\left(1-q \rho^{\prime} \rho\right)^{-1}$.

Assumption 2.13 is straightforward to check, and holds when $p \leq 0$ for virtually all models in the literature, with the exception of correlation risk (cf. Buraschi, Porchia, and Trojani (2010)).

Set $\left(\mathcal{F}_{t}^{R, Y}\right)_{t \geq 0}$ as the right continuous envelope of the filtration generated by $(R, Y)$. For any admissible strategy $H$ with respect to this filtration, the corresponding risky weight $\pi=H S / X$ is an adapted, $R$-integrable process $\left(\pi_{t}\right)_{t \geq 0}^{1 \leq i \leq d}$, and satisfies the relation

$$
\frac{d X_{t}^{\pi}}{X_{t}^{\pi}}=r\left(Y_{t}\right) d t+\pi_{t}^{\prime} d R_{t}
$$

In this Markovian setting, the value function for the horizon $T \in \mathbb{R}_{+}$is given by:

$$
u^{0, T}=u^{T}(t, x, y)=\sup _{\pi \text { admissible }} \mathbb{E}^{\mathbb{P}}\left[\left(X_{T}^{\pi}\right)^{p} / p \mid X_{t}=x, Y_{t}=y\right], \quad \text { for } t \in[0, T] .
$$

These utility maximization problems are studied at all horizons under the following assumption:

Assumption 2.14. There exist $\left(\hat{v}, \lambda_{c}\right)$ such that $\hat{v} \in C^{2}(E), \hat{v}>0$, and solves the equation:

$$
\mathcal{L} v+c v=\lambda v, \quad y \in E
$$

where

$$
\mathcal{L}:=\frac{1}{2} A \partial_{y y}^{2}+B \partial_{y} ; \quad B:=b-q \Upsilon^{\prime} \Sigma^{-1} \mu .
$$

Also, for the $y_{0} \in E$ in Assumption 2.9P

$$
\begin{array}{rlrl}
\int_{\alpha}^{y_{0}} \frac{1}{\hat{v}^{2} A \hat{m}(y)} d y & =\infty & \int_{y_{0}}^{\beta} \frac{1}{\hat{v}^{2} A \hat{m}(y)} d y & =\infty, \\
\int_{\alpha}^{\beta} \hat{v}^{2} \hat{m}(y) d y & =1 & \int_{\alpha}^{\beta} \hat{v} \hat{m}(y) d y<\infty,
\end{array}
$$

where

$$
\hat{m}(y):=\frac{1}{A(y)} \exp \left(\int_{y_{0}}^{y} \frac{2 B(z)}{A(z)} d z\right) .
$$

\footnotetext{
${ }^{3}$ Any $y_{0} \in E$ suffices. This $y_{0}$ is chosen to align $m$ with $\hat{m}$.
} 
Remark 2.15. If $\hat{v}>0$ satisfies (2.12), (2.14), the inequality in (2.15), then $\int_{\alpha}^{\beta} \hat{v}^{2} \hat{m}(y) d y=1$ is equivalent to $\int_{\alpha}^{\beta} \hat{v}^{2} \hat{m}(y) d y<\infty$, up to a renormalization of $\hat{v}$. We assume that the integral equals one only for convenience of notation.

Assumption 2.14 is interpreted as follows. Equation (2.12) is the ergodic HJB equation, which controls the long-run limit of the utility maximization problem (cf. Guasoni and Robertson (2009) Theorem 7 and Section 2.2.1). Its solution $\hat{v}$ is related to the finite-horizon value functions $u^{T}$ by $u^{T}(x, y, 0) \sim\left(x^{p} / p\right)\left(e^{\lambda T} \hat{v}(y)\right)^{\delta}$. Thus, assuming that (2.12) has a solution guarantees that the long-run optimization problem is well posed. The presence of $\delta$ reflects the power transformation of Zariphopoulou (2001), which allows to write the ergodic HJB equation in the linear form (2.12).

To understand the meaning of (2.14) and (2.15), define the operator:

$$
\hat{\mathcal{L}}=\frac{1}{2} \sum_{i, j=1}^{d+1} \widetilde{A}_{i j}(\xi) \frac{\partial^{2}}{\partial \xi_{i} \partial \xi_{j}}+\sum_{i=1}^{d+1} \hat{b}_{i}(\xi) \frac{\partial}{\partial \xi_{i}}, \quad \hat{b}=\left(\begin{array}{c}
-q \bar{\rho}^{\prime} \sigma^{\prime} \Sigma^{-1}\left(\mu+\delta \Upsilon \frac{\hat{v}_{y}}{\hat{v}}\right) \\
B+A \frac{\hat{v}_{y}}{\hat{v}}
\end{array}\right)
$$

where $\widetilde{A}$ is the same as in (2.8). Condition (2.14) in Assumption 2.14 implies that the martingale problem for $\hat{\mathcal{L}}$ on $\mathbb{R}^{d} \times E$ has a unique solution $\left(\hat{\mathbb{P}}^{\xi}\right)_{\xi \in \mathbb{R}^{d} \times E}$ and that $\hat{\mathbb{P}}^{\xi}$ is equivalent to $\mathbb{P}^{\xi}$ (see Lemma 4.3 below). The family $\left(\hat{\mathbb{P}}^{\xi}\right)_{\xi \in \mathbb{R}^{d} \times E}$ is called the long-run probability. Girsanov's theorem in turn implies that the following stochastic differential equation has a unique weak solution starting at $\xi$ under $\hat{\mathbb{P}}^{\xi}$ :

$$
\begin{aligned}
& d R_{t}=\frac{1}{1-p}\left(\mu+\delta \Upsilon \frac{\hat{v}_{y}}{\hat{v}}\right)\left(Y_{t}\right) d t+\sigma\left(Y_{t}\right) d \hat{Z}_{t} \\
& d Y_{t}=\left(B+A \frac{\hat{v}_{y}}{\hat{v}}\right)\left(Y_{t}\right) d t+a\left(Y_{t}\right) d \hat{W}_{t}
\end{aligned}
$$

Here, $(\hat{B}, \hat{W})$ is a $d+1$ dimensional Brownian Motions under $\hat{\mathbb{P}} \xi$, and $\hat{Z}=\rho \hat{B}+\bar{\rho} \hat{W}$. Conditions (2.14) and (2.15) imply that $Y$ is ergodic under $\left(\hat{\mathbb{P}}^{\xi}\right)_{\xi \in \mathbb{R}^{d} \times E}$ (Lemma 4.2 below, and Section 4.1 for a precise definition of ergodicity). This property drives the long-run asymptotics of the projected densities in (2.5).

A simple criterion to check Assumption 2.14 is the following:

Proposition 2.16. Let Assumptions 2.8 and 2.13 hold. If $c$ in (2.9) and $\hat{m}$ in (2.16) satisfy:

$$
\begin{aligned}
& \int_{\alpha}^{\beta} \hat{m}(y) d y<\infty, \\
& \lim _{y \downarrow \alpha} c(y)=\lim _{y \uparrow \beta} c(y)=-\infty .
\end{aligned}
$$

Then, Assumption 2.14 holds.

Remark 2.17. If the interest rate $r$ is bounded from below, and $p<0$, (2.20) states that the squared norm of the vector of risk premia $\sigma^{-1} \mu$ goes to $\infty$ at the boundary of the state space $E$. 
Assumption 2.14 guarantees that (see Proposition 4.7 below) at all finite horizons $T$, the value functions $u^{T}$ in (2.11) can be represented as $u^{T}(t, x, y)=\left(x^{p} / p\right)\left(v^{T}(t, y)\right)^{\delta}$ for $(t, x, y) \in[0, T] \times$ $\mathbb{R}_{+} \times E$, where $v^{T}$ is a strictly positive classical solution to the linear parabolic PDE:

$$
\begin{array}{ll}
\partial_{t} v+\mathcal{L} v+c v=0, & (t, y) \in(0, T) \times E, \\
v(T, y)=1, & y \in E .
\end{array}
$$

Moreover, the optimal portfolio for the horizon $T$ is (all functions are evaluated at $\left(t, Y_{t}\right)$ ):

$$
\pi^{T}=\frac{1}{1-p} \Sigma^{-1}\left(\mu+\delta \Upsilon \frac{v_{y}^{T}}{v^{T}}\right) .
$$

Thus, the wealth process corresponding to this portfolio leads to the optimal terminal wealth $X_{T}^{\pi^{T}}$, which in turn defines the probability $\mathbb{P}^{T, y}$ by (2.4). Understanding the convergence of $d \mathbb{P}^{T, y} /\left.d \mathbb{P}^{y}\right|_{\mathcal{F}_{t}}$ is key to go beyond the abstract version of the turnpike. To this end, observe from (2.18) that the portfolio:

$$
\hat{\pi}=\frac{1}{1-p} \Sigma^{-1}\left(\mu+\delta \Upsilon \frac{\hat{v}_{y}}{\hat{v}}\right)
$$

evaluated at $\left(t, Y_{t}\right)$, is optimal for logarithmic utility under the probability $\hat{\mathbb{P}}^{y}$. This fact suggests that the conditional densities of $\hat{\mathbb{P}}^{y}$ are natural candidates for the limits of the conditional densities $d \mathbb{P}^{T, y} /\left.d \mathbb{P}^{y}\right|_{\mathcal{F}_{t}}$. Combined with the ergodicity of $Y$ under $\left(\hat{\mathbb{P}}^{y}\right)_{y \in E}$ the next result follows:

Lemma 2.18. Let Assumptions [2.8, 2.9, 2.13, and 2.14 hold. Then, for all $y \in E$ and $t, \varepsilon>0$ :

$$
\lim _{T \rightarrow \infty} \hat{\mathbb{P}}^{y}\left(\left|\frac{d \mathbb{P}^{T, y}}{d \hat{\mathbb{P}}^{y}}\right|_{\mathcal{F}_{t}}-1 \mid \geq \epsilon\right)=0 .
$$

This result essentially allows to replace $\mathbb{P}^{T, y}$ in Theorem 2.5 with $\hat{\mathbb{P}}^{y}$. Then, the classic turnpike follows from the equivalence of $\hat{\mathbb{P}}^{y}$ and $\mathbb{P}^{y}$ (cf. Lemma 4.3, part (ii)):

Theorem 2.19 (Classic Turnpike). Let Assumptions 2.1 - 2.4, 2.8, 2.9, 2.13 and 2.14 hold. Then, for all $y \in E, 0 \neq p<1$ and $\epsilon, t>0$ :

a) $\lim _{T \rightarrow \infty} \mathbb{P}^{y}\left(\sup _{u \in[0, t]}\left|r_{u}^{T}-1\right| \geq \epsilon\right)=0$,

b) $\lim _{T \rightarrow \infty} \mathbb{P}^{y}\left(\left[\Pi^{T}, \Pi^{T}\right]_{t} \geq \epsilon\right)=0$.

Abstract and classic turnpikes compare the finite-horizon optimal portfolio of a generic utility to that of its CRRA benchmark at the same finite horizon. By contrast, the explicit turnpike, discussed next, uses as a benchmark the long horizon limit of the optimal CRRA portfolio.

This result has two main implications: first, and most importantly, it shows that the two approximations of replacing a generic utility with a power, and a finite horizon problem with its long-run limit, lead to small errors as the horizon becomes large. Second, this theorem has a nontrivial

\footnotetext{
${ }^{4}$ Since $R_{0}=0$ by assumption, $\mathbb{P}^{\xi}$ with $\xi=(0, y)$ is denoted as $\mathbb{P}^{y}$. The same convention applies to $\hat{\mathbb{P}}^{\xi}$.
} 
statement even for $U$ in the CRRA class: in this case, it states that the optimal finite-horizon portfolio converges to the long-run optimal portfolio, identified as a solution to the ergodic HJB equation (2.12).

To state the explicit turnpike, define, in analogy to 2.3, the ratio of optimal wealth processes relative to the long-run benchmark, and their stochastic logarithms as:

$$
\hat{r}_{u}^{T}:=\frac{X_{u}^{1, T}}{\hat{X}_{u}}, \quad \hat{\Pi}_{u}^{T}:=\int_{0}^{u} \frac{d \hat{r}_{v}^{T}}{\hat{r}_{v-}^{T}}, \quad \text { for } u \in[0, T],
$$

where $\hat{X}$ is the wealth process of the long-run portfolio $\hat{\pi}$.

Theorem 2.20 (Explicit Turnpike). Under the assumptions of Theorem 2.19, for any $y \in E$, $\epsilon, t>0$ and $0 \neq p<1$ :

a) $\lim _{T \rightarrow \infty} \mathbb{P}^{y}\left(\sup _{u \in[0, t]}\left|\hat{r}_{u}^{T}-1\right| \geq \epsilon\right)=0$,

b) $\lim _{T \rightarrow \infty} \mathbb{P}^{y}\left(\left[\hat{\Pi}^{T}, \hat{\Pi}^{T}\right]_{t} \geq \epsilon\right)=0$.

If $U$ is in CRRA class, GROWTH is not needed for the above convergence.

2.3. Applications. Before proving the main results, we offer two examples of their significance. We begin with an application to target-date mutual funds and the social planner problem.

Example 2.21. Consider several investors, who differ in their initial capitals $\left(x_{i}\right)_{i=1}^{n}$ and risk aversions $\left(\gamma_{i}\right)_{i=1}^{n}$, but share the same long horizon $T$. Suppose that they do not invest independently, but rather pool their wealth into a common fund, delegate a manager to invest it, and then collect the proceeds on their respective capitals under the common investment strategy. This setting is typical of target-date retirement funds, in which savings from a diverse pool of participants are managed according to a single strategy, characterized by the common horizon $T$.

Suppose the manager invests as to maximize a weighted sum of the investors' expected utilities, thereby solving the problem

$$
\max _{X \in \mathcal{X}^{T}} \sum_{i=1}^{n} w_{i} \mathbb{E}^{\mathbb{P}}\left[\frac{\left(x_{i} X_{T}\right)^{1-\gamma_{i}}}{1-\gamma_{i}}\right]
$$

for some $\left(w_{i}\right)_{i=1}^{n}>0$. By homogeneity and linearity, this problem is equivalent to maximizing the expected value $\mathbb{E}^{\mathbb{P}}\left[U\left(X_{T}\right)\right]$ of the master utility functions:

$$
U(x)=\sum_{i=1}^{n} \tilde{w}_{i} \frac{x^{1-\gamma_{i}}}{1-\gamma_{i}} \quad \text { where } \tilde{w}_{i}=w_{i} x_{i}^{1-\gamma_{i}} .
$$

Thus, the fund manager is akin to a social planner, who ponders the welfare of various investors according to the weights $\tilde{w}_{i}$. The question is how these weights affect the choice of the common fund's strategy, if the horizon is distant, as for most retirement funds.

\footnotetext{
${ }^{5}$ If a logarithmic investor is present $\left(\gamma_{i}=1\right.$ for some $\left.i\right)$, a constant is added to $U(x)$, and the stated equivalence remains valid.
} 
While this problem is intractable for a fixed horizon $T$, turnpike theorems offer a crisp solution in the long run limit. Indeed, the master utility function satisfies Assumption 2.1 with $\gamma=1-p=$ $\min _{1 \leq i \leq n} \gamma_{i}$. Thus, for any market that satisfies the additional Assumptions 2.2 2.4, it is optimal for the fund manager to act on behalf of the least risk-averse investor.

The implication is that most or nearly all fund participants will find that the fund takes more risk than they would like, regardless of the welfare weights $\tilde{w}_{i}$ (provided that they are strictly positive). The result holds irrespective of market completeness or independence of returns, and indicates that a social planner objective is ineffective in choosing a portfolio that balances the needs or investors with different preferences.

Note that this result points in the same direction as the ones of Benninga and Mavshar (2000) and Cvitanic and Malamud (2008), with the crucial difference that prices are endogenous in their models, while they are exogenous in our setting. Finally, the result should be seen in conjunction with the classical numeraire property of the log-optimal portfolio, whereby the wealth process of the logarithmic investor becomes arbitrarily larger than any other wealth process. In spite of this property, the fund manager does not choose the log-optimal strategy, but the one optimal for the least risk-averse investor.

The next example is more technical. In the model that follows, returns of risky assets have constant volatility, but their drift is an independent Ornstein-Uhlenbeck process. Such independence entails that optimal CRRA portfolios are myopic, as in assumption i) of Corollary 2.7. By contrast, the time-varying drift makes returns dependent over time, hence assumption $i i$ ) of the same corollary does not hold. As a result, the proof of Corollary 2.7 fails, because it requires that $d \mathbb{P}^{T, y} /\left.d \mathbb{P}^{y}\right|_{\mathcal{F}_{t}}$ is constant with respect to $T$, which is not the case here. Yet, both the classic and the explicit turnpikes hold in this model, in the form of Theorems 2.19] and 2.20. Of course, these results depend on the ergodicity of the diffusion $Y$.

Example 2.22. Consider the diffusion model:

$$
d R_{t}=Y_{t} d t+d Z_{t} \quad \text { and } \quad d Y_{t}=-Y_{t} d t+d W_{t}
$$

where the correlation between $Z$ and $W$ is $\rho=0$ and the safe rate $r>0$. Clearly, Assumptions 2.8 and 2.9 hold. Furthermore, for $p<0$ Assumption 2.13, as well as the hypotheses of Proposition 2.16 are met and hence Assumption 2.14 holds as well, yielding the results of Theorems 2.19 and 2.20. The optimal portfolio for a CRRA investor is a myopic portfolio $\pi_{t}^{T}=Y_{t} /(1-p)$; see (2.22). However, the conditional density $d \mathbb{P}^{T, y} /\left.d \mathbb{P}^{y}\right|_{\mathcal{F}_{t}}$ depends on the horizon $T$. Indeed, it follows from Proposition 4.6 below that

$$
\left.\frac{d \mathbb{P}^{T, y}}{d \mathbb{P}^{y}}\right|_{\mathcal{F}_{t}}=\mathcal{E}\left(\int \frac{v_{y}^{T}\left(s, Y_{s}\right)}{v^{T}\left(s, Y_{s}\right)} d W_{s}-q \int_{0}^{\cdot} Y_{s} d Z_{s}\right)_{t},
$$


where $v^{T}$ satisfies the HJB equation $\partial_{t} v+\frac{1}{2} \partial_{y y}^{2} v-y \partial_{y} v+\left(r p-\frac{q}{2} y^{2}\right) v=0$ with $v(T, y)=1$. The above conditional density is independent of $T$ only if $g^{T}(t, y):=v_{y}^{T}(t, y) / v^{T}(t, y)$ is independent of $T$ for any fixed $(t, y)$. It can be shown that $v^{T}$ is smooth, and not just twice continuously differentiable, in the state variable $y$, and hence $g^{T}$ satisfies $\partial_{t} g+\frac{1}{2} \partial_{y y}^{2} g+(g-y) \partial_{y} g-g-q y=0$ with $g(T, y)=0$. If $g^{T}$ was independent of $T, 0$ should be a solution to the previous equation. However, this is clearly not the case for $q \neq 0$.

\section{Proof of the Abstract Turnpike}

This section contains the results leading to the abstract version of the turnpike theorem. The proof proceeds through two main steps:

i) Establish that optimal payoffs for the generic utility converge to their CRRA counterparts;

ii) Obtain from the convergence of optimal payoffs the convergence of wealth processes.

3.1. Convergence of optimal payoffs. First, note that Assumption 2.3 implies the existence of a deflator, that is a strictly positive process $Y$ such that $Y X$ is a (nonnegative) supermartingale for all $X \in \mathcal{X}^{T}$ and $T>0$. Condition (GROWTH) entails that $\lim _{T \rightarrow \infty} \mathbb{E}\left[Y_{T}\right]=0$ for any such deflator $Y$. In this section, the capital letter $Y$ is used for deflators, while in the section on diffusion models it denotes the state variable. Recall a result from Karatzas and Žitković (2003):

Theorem 3.1 (Karatzas-Žitković). Under Assumptions 2.1 - 2.4, the optimal payoffs are

$$
X_{T}^{i, T}=I^{i}\left(y^{i, T} Y_{T}^{i, T}\right), \quad i=0,1, T>0
$$

where $I^{0}$ is the inverse function of $x^{p-1}, I^{1}$ is the inverse function of $U^{\prime}(x)$, the positive constant $y^{i, T}$ is the Lagrangian multiplier, and $Y^{i, T}$ is some supermartingale deflator. Moreover,

$$
y^{i, T}=\mathbb{E}^{\mathbb{P}}\left[\left(U^{i}\right)^{\prime}\left(X_{T}^{i, T}\right) X_{T}^{i, T}\right] \geq \mathbb{E}^{\mathbb{P}}\left[\left(U^{i}\right)^{\prime}\left(X_{T}^{i, T}\right) X_{T}\right], \quad i=0,1, T>0,
$$

for any $X \in \mathcal{X}^{T}$. Here $U^{0}(x)=x^{p} / p$ and $U^{1}(x)=U(x)$.

Remark 3.2.

i) It follows from (3.1) and the Inada condition that $X_{T}^{i, T}>0 \mathbb{P}$-a.s. for $i=0,1$ and $T \geq 0$. Since $X^{i, T}$ is a nonnegative $\mathbb{Q}^{T}$-supermartingale and $\mathbb{Q}^{T}$ is equivalent to $\mathbb{P}$, it follows that $X_{t}^{i, T}>0 \mathbb{P}$-a.s. for $0 \leq t \leq T$.

ii) Condition (GROWTH entails that $\lim _{T \rightarrow \infty} \mathbb{E}^{\mathbb{P}}\left[Y_{T}^{i, T}\right]=0$ for $i=0,1$ and $\lim _{T \rightarrow \infty} \mathbb{P}^{T}\left(S_{T}^{0} \geq\right.$ $N)=1$ for any $N>0$.

iii) Recall the probability measure $\mathbb{P}^{T}$ defined in (2.4). The optimal wealth process $X^{0, T}$ has the numéraire property under $\mathbb{P}^{T}$, i.e. $\mathbb{E}^{\mathbb{P}^{T}}\left[X_{T} / X_{T}^{0, T}\right] \leq 1$ for any $X \in \mathcal{X}^{T}$. This claim follows from $\mathbb{E}^{\mathbb{P}}\left[\left(X_{T}^{0, T}\right)^{p}\left(X_{T} / X_{T}^{0, T}-1\right)\right] \leq 0$, obtained from (3.2), and switching the expectation from $\mathbb{P}$ to $\mathbb{P}^{T}$. 
Both $X_{T}^{0, T}$ and $X_{T}^{1, T}$ will be shown to be unbounded as $T \rightarrow \infty$. However, the main result of this subsection, Lemma 3.8, shows that their ratio at the horizon $T$, given by $r_{T}^{T}$ from (2.3) satisfies $\lim _{T \rightarrow \infty} \mathbb{E}^{\mathbb{P}^{T}}\left[\left|r_{T}^{T}-1\right|\right]=0$. Lemma 3.8 will be the culmination of a series of auxiliary results. Assumptions 2.1-2.4 are enforced in the rest of this subsection.

Lemma 3.3. It holds that

$$
\lim _{T \rightarrow \infty} \mathbb{P}^{T}\left(X_{T}^{0, T} \geq N\right)=1, \quad \text { for any } N>0
$$

Proof. It suffices to prove $\lim \sup _{T \rightarrow \infty} \mathbb{P}^{T}\left(X_{T}^{0, T}<N\right)=0$ for each fixed $N$. To this end, the numéraire property of $X^{0, T}$ under $\mathbb{P}^{T}$ implies that:

$$
1 \geq \mathbb{E}^{\mathbb{P}^{T}}\left[\frac{S_{T}^{0}}{X_{T}^{0, T}}\right] \geq \mathbb{E}^{\mathbb{P}^{T}}\left[\frac{S_{T}^{0}}{X_{T}^{0, T}} 1_{\left\{X_{T}^{0, T}<N, S_{T}^{0} \geq \tilde{N}\right\}}\right] \geq \frac{\tilde{N}}{N} \mathbb{P}^{T}\left(X_{T}^{0, T}<N, S_{T}^{0} \geq \tilde{N}\right)
$$

for any positive constant $\tilde{N}$. As a result, $\mathbb{P}^{T}\left(X_{T}^{0, T}<N, S_{T}^{0} \geq \tilde{N}\right) \leq N / \tilde{N}$. Combining the last inequality with Remark 3.2 item ii), it follows that

$$
\limsup _{T \rightarrow \infty} \mathbb{P}^{T}\left(X_{T}^{0, T}<N\right) \leq \limsup _{T \rightarrow \infty} \mathbb{P}^{T}\left(X_{T}^{0, T}<N, S_{T}^{0} \geq \tilde{N}\right)+\lim _{T \rightarrow \infty} \mathbb{P}^{T}\left(S_{T}^{0}<\tilde{N}\right) \leq \frac{N}{\tilde{N}}
$$

Then, the statement follows since $\tilde{N}$ is chosen arbitrarily.

Recall the Lagrangian multipliers $y^{i, T}, i=0,1$, from Theorem 3.1. The following result presents the asymptotic behavior of $y^{0, T} / y^{1, T}$ as $T \rightarrow \infty$.

\section{Lemma 3.4.}

$$
\liminf _{T \rightarrow \infty} \frac{y^{0, T}}{y^{1, T}} \geq 1
$$

Proof. The statement will be proved separately for $p=0, p \in(0,1)$, and $p<0$. Throughout this proof, in order to ease notation, we set $\alpha_{T}=y^{1, T}, Y_{T}=Y_{T}^{1, T}, \widetilde{Y}_{T}=Y_{T}^{0, T}, X_{T}=X_{T}^{1, T}, \widetilde{X}_{T}=X_{T}^{0, T}$, and $I=\left(U^{\prime}\right)^{-1}$. All expectations are under $\mathbb{P}$. Observe first that

$$
\lim _{y \downarrow 0} I(y) y^{\frac{1}{1-p}}=1 .
$$

Indeed, set $x=I(y)$, hence $x \uparrow \infty$ as $y \downarrow 0$. Then the convergence above follows from (CONV) via

$$
\frac{I(y)}{y^{\frac{1}{p-1}}}=\frac{I\left(U^{\prime}(x)\right)}{\left(U^{\prime}(x)\right)^{\frac{1}{p-1}}}=\frac{x}{\left(U^{\prime}(x)\right)^{\frac{1}{p-1}}}=\left(\frac{x^{p-1}}{U^{\prime}(x)}\right)^{\frac{1}{p-1}} \rightarrow 1, \quad \text { as } y \downarrow 0 .
$$


Case $p=0$ : It follows from (3.3) that for any $\epsilon>0$ there exists $\delta>0$ such that $1-\epsilon \leq y I(y) \leq 1+\epsilon$ for $y<\delta$. Then (3.1) and (3.2) imply

$$
\begin{aligned}
1 & =\mathbb{E}\left[Y_{T} I\left(\alpha_{T} Y_{T}\right)\right]=\mathbb{E}\left[Y_{T} I\left(\alpha_{T} Y_{T}\right) 1_{\left\{\alpha_{T} Y_{T}<\delta\right\}}+Y_{T} I\left(\alpha_{T} Y_{T}\right) 1_{\left\{\alpha_{T} Y_{T} \geq \delta\right\}}\right] \\
& \leq \frac{1+\epsilon}{\alpha_{T}} \mathbb{P}\left(\alpha_{T} Y_{T}<\delta\right)+I(\delta) \mathbb{E}\left[Y_{T} 1_{\left\{\alpha_{T} Y_{T} \geq \delta\right\}}\right] \\
& \leq \frac{1+\epsilon}{\alpha_{T}}+I(\delta) \mathbb{E}\left[Y_{T}\right]
\end{aligned}
$$

where the first inequality follows because $I$ is decreasing. Now, the previous inequality combined with Remark 3.2 item ii) implies that

$$
1 \leq \liminf _{T \rightarrow \infty} \frac{1+\epsilon}{\alpha_{T}}
$$

from which the statement follows since for $p=0, y^{0, T}=1$ and $\epsilon$ is chosen arbitrarily.

Case $p \in(0,1)$ : It follows from (CONV) that for any $\epsilon>0$ there exists $M>0$ such that $1-\epsilon \leq$ $\overline{U^{\prime}(x) x^{1-p} \leq 1}+\epsilon$ for $x \geq M$. Then (3.2) implies that

$$
\begin{aligned}
1 & =\frac{1}{\alpha_{T}} \mathbb{E}\left[U^{\prime}\left(X_{T}\right) X_{T}\right]=\frac{1}{\alpha_{T}} \mathbb{E}\left[U^{\prime}\left(X_{T}\right) X_{T}^{1-p} X_{T}^{p} 1_{\left\{X_{T} \geq M\right\}}\right]+\frac{1}{\alpha_{T}} \mathbb{E}\left[U^{\prime}\left(X_{T}\right) X_{T} 1_{\left\{X_{T} \leq M\right\}}\right] \\
& \leq \frac{1+\epsilon}{\alpha_{T}} \mathbb{E}\left[X_{T}^{p} 1_{\left\{X_{T} \geq M\right\}}\right]+\frac{1}{\alpha_{T}} \mathbb{E}\left[U^{\prime}\left(X_{T}\right) X_{T} 1_{\left\{X_{T} \leq M\right\}}\right] .
\end{aligned}
$$

Note that $\left(1 / \alpha_{T}\right) \mathbb{E}\left[U^{\prime}\left(X_{T}\right) X_{T} 1_{\left\{X_{T} \leq M\right\}}\right]=\mathbb{E}\left[Y_{T} X_{T} 1_{\left\{X_{T} \leq M\right\}}\right] \leq M \mathbb{E}\left[Y_{T}\right] \rightarrow 0$, as $T \rightarrow \infty$. Therefore

$$
\frac{1}{1+\epsilon} \leq \liminf _{T \rightarrow \infty} \frac{1}{\alpha_{T}} \mathbb{E}\left[X_{T}^{p} 1_{\left\{X_{T} \geq M\right\}}\right] \leq \liminf _{T \rightarrow \infty} \frac{1}{\alpha_{T}} \mathbb{E}\left[X_{T}^{p}\right] \leq \liminf _{T \rightarrow \infty} \frac{1}{\alpha_{T}} \mathbb{E}\left[\widetilde{X}_{T}^{p}\right],
$$

where the third inequality follows from the optimality of $\tilde{X}=X^{0, T}$ for $\sup _{x \in \mathcal{X}^{T}} \mathbb{E}\left[X_{T}^{p} / p\right]$. Note that $y^{0, T}=\mathbb{E}\left[\widetilde{X}_{T}^{p}\right]$. The statement follows from the previous inequality since $\epsilon$ is chosen arbitrarily.

Case $p<0$ : For any $\epsilon>0$ there exists $\delta>0$ such that $1-\epsilon \leq I(y) y^{\frac{1}{1-p}} \leq 1+\epsilon$ for $y<\delta$. Then (3.1) and (3.2) yield (recall $q=p /(p-1)$ is the conjugate exponent to $p$ )

$$
\begin{aligned}
1 & =\mathbb{E}\left[Y_{T} I\left(\alpha_{T} Y_{T}\right)\right]=\mathbb{E}\left[Y_{T} I\left(\alpha_{T} Y_{T}\right) 1_{\left\{\alpha_{T} Y_{T}<\delta\right\}}\right]+\mathbb{E}\left[Y_{T} I\left(\alpha_{T} Y_{T}\right) 1_{\left\{\alpha_{T} Y_{T} \geq \delta\right\}}\right] \\
& \leq \frac{1+\epsilon}{\alpha_{T}^{1-p}} \mathbb{E}\left[Y_{T}^{q} 1_{\left\{\alpha_{T} Y_{T}<\delta\right\}}\right]+\mathbb{E}\left[Y_{T} I\left(\alpha_{T} Y_{T}\right) 1_{\left\{\alpha_{T} Y_{T} \geq \delta\right\}}\right] .
\end{aligned}
$$

Since $\mathbb{E}\left[Y_{T} I\left(\alpha_{T} Y_{T}\right) 1_{\left\{\alpha_{T} Y_{T} \geq \delta\right\}}\right] \leq I(\delta) \mathbb{E}\left[Y_{T}\right] \rightarrow 0$, as $T \rightarrow \infty$, the inequality in the last line yields

$$
\frac{1}{1+\epsilon} \leq \liminf _{T \rightarrow \infty} \frac{1}{\alpha_{T}^{\frac{1}{1-p}}} \mathbb{E}\left[Y_{T}^{q} 1_{\left\{\alpha_{T} Y_{T}<\delta\right\}}\right] \leq \liminf _{T \rightarrow \infty} \frac{1}{\alpha_{T}^{\frac{1}{1-p}}} \mathbb{E}\left[Y_{T}^{q}\right] .
$$

The $1-p^{t h}$ power on both sides of the previous inequality gives

$$
\left(\frac{1}{1+\epsilon}\right)^{1-p} \leq \liminf _{T \rightarrow \infty} \frac{1}{\alpha_{T}} \mathbb{E}\left[Y_{T}^{q}\right]^{1-p} \leq \liminf _{T \rightarrow \infty} \frac{1}{\alpha_{T}} \mathbb{E}\left[\widetilde{X}_{T}^{p}\right],
$$


from which the statement follows. Since $p<0$, the second inequality above follows from

$$
\frac{1}{p} \mathbb{E}\left[\widetilde{X}_{T}^{p}\right]=\frac{1}{p} \mathbb{E}\left[\widetilde{Y}_{T}^{q}\right]^{1-p} \leq \frac{1}{p} \mathbb{E}\left[Y_{T}^{q}\right]^{1-p},
$$

where the equality holds due to the duality for power utility and the inequality follows from the optimality of $\widetilde{Y}$ for the dual problem which minimizes $\mathbb{E}\left[-\mathcal{Y}_{T}^{q} / q\right]$ among all supermartingale deflators $\mathcal{Y}$.

The previous two lemmas combined describe the asymptotic behavior of $X_{T}^{1, T}$ and $\mathfrak{R}\left(X_{T}^{1, T}\right)$ where $\mathfrak{R}$ is given in (2.1).

Lemma 3.5. It holds that

$$
\lim _{T \rightarrow \infty} \mathbb{P}^{T}\left(X_{T}^{1, T} \geq N\right)=1, \quad \text { for any } N>0
$$

Hence

$$
\lim _{T \rightarrow \infty} \mathbb{P}^{T}\left(\left|\Re\left(X_{T}^{1, T}\right)-1\right| \geq \epsilon\right)=0, \quad \text { for any } \epsilon>0 .
$$

Proof. It follows from Lemma 3.4 and (3.2) that

$$
2 \geq \frac{y^{1, T}}{y^{0, T}} \geq \frac{\mathbb{E}^{\mathbb{P}}\left[X_{T}^{0, T} U^{\prime}\left(X_{T}^{1, T}\right)\right]}{\mathbb{E}^{\mathbb{P}}\left[\left(X_{T}^{0, T}\right)^{p}\right]}=\mathbb{E}^{\mathbb{P}^{T}}\left[\frac{U^{\prime}\left(X_{T}^{1, T}\right)}{\left(X_{T}^{0, T}\right)^{p-1}}\right], \quad \text { for sufficiently large } T .
$$

Combining the previous inequality with Lemma 3.3, the first statement follows. Indeed, for any given $M$ and $N$, on the set $\left\{X_{T}^{1, T} \leq N ; X_{T}^{0, T} \geq M\right\},\left(X_{T}^{0, T}\right)^{1-p} \geq M^{1-p}$ and $U^{\prime}\left(X_{T}^{1, T}\right) \geq U^{\prime}(N)$, therefore

$$
2 \geq \mathbb{E}^{\mathbb{P}^{T}}\left[\frac{U^{\prime}\left(X_{T}^{1, T}\right)}{\left(X_{T}^{0, T}\right)^{p-1}} 1_{\left\{X_{T}^{1, T} \leq N ; X_{T}^{0, T} \geq M\right\}}\right] \geq U^{\prime}(N) M^{1-p} \mathbb{P}^{T}\left(X_{T}^{1, T} \leq N ; X_{T}^{0, T} \geq M\right) .
$$

Hence,

$$
\mathbb{P}^{T}\left(X_{T}^{1, T} \leq N\right) \leq \mathbb{P}^{T}\left(X_{T}^{1, T} \leq N ; X_{T}^{0, T} \geq M\right)+\mathbb{P}^{T}\left(X_{T}^{0, T} \leq M\right) \leq \frac{2}{U^{\prime}(N) M^{1-p}}+\mathbb{P}^{T}\left(X_{T}^{0, T} \leq M\right) .
$$

Letting first $T \rightarrow \infty$ and then $M \rightarrow \infty$ in the previous inequality, the first statement follows.

We move to the proof of the second statement. For any $\varepsilon>0$, due to (CONV), there exists a sufficiently large $N_{\varepsilon}$ such that $|\mathfrak{R}(x)-1|<\varepsilon$ for any $x>N_{\varepsilon}$. As a result, $\mathbb{P}^{T}\left(\left|\Re\left(X_{T}^{1, T}\right)-1\right| \geq\right.$ $\left.\varepsilon, X_{T}^{1, T}>N_{\varepsilon}\right)=0$. Combining the previous identity with $\lim _{T \rightarrow \infty} \mathbb{P}^{T}\left(X_{T}^{1, T} \leq N_{\varepsilon}\right)=0$, the second statement follows.

We continue with the following result, which is crucial for the proof of Lemma 3.8 later on. Recall that $r^{T}$ is given in (2.3).

Lemma 3.6. It holds that

$$
\lim _{T \rightarrow \infty} \mathbb{E}^{\mathbb{P}^{T}}\left[\left|1-\mathfrak{R}\left(X_{T}^{1, T}\right)\left(r_{T}^{T}\right)^{p-1}\right|\left|r_{T}^{T}-1\right|\right]=0
$$


Proof. To ease notation, set $\mathfrak{R}_{T}=\mathfrak{R}\left(X_{T}^{1, T}\right)$ and $r_{T}=r_{T}^{T}$. It follows from (3.2) that the two inequalities $\mathbb{E}^{\mathbb{P}}\left[\left(X_{T}^{0, T}\right)^{p-1}\left(X_{T}^{1, T}-X_{T}^{0, T}\right)\right] \leq 0$ and $\mathbb{E}^{\mathbb{P}}\left[U^{\prime}\left(X_{T}^{1, T}\right)\left(X_{T}^{0, T}-X_{T}^{1, T}\right)\right] \leq 0$ hold. Summing these two inequalities, it follows that

$$
\begin{aligned}
0 & \geq \mathbb{E}^{\mathbb{P}}\left[\left(\left(X_{T}^{0, T}\right)^{p-1}-U^{\prime}\left(X_{T}^{1, T}\right)\right)\left(X_{T}^{1, T}-X_{T}^{0, T}\right)\right], \\
& =\mathbb{E}^{\mathbb{P}}\left[\left(X_{T}^{0, T}\right)^{p-1}\left(1-\frac{U^{\prime}\left(X_{T}^{1, T}\right)}{\left(X_{T}^{1, T}\right)^{p-1}} \frac{\left(X_{T}^{1, T}\right)^{p-1}}{\left(X_{T}^{0, T}\right)^{p-1}}\right)\left(X_{T}^{1, T}-X_{T}^{0, T}\right)\right], \\
& =\mathbb{E}^{\mathbb{P}}\left[\left(X_{T}^{0, T}\right)^{p}\left(1-\mathfrak{R}_{T} r_{T}^{p-1}\right)\left(r_{T}-1\right)\right] .
\end{aligned}
$$

After changing to the measure $\mathbb{P}^{T}$, the previous inequality reads

$$
\mathbb{E}^{\mathbb{P}^{T}}\left[\left(1-\mathfrak{R}_{T} r_{T}^{p-1}\right)\left(r_{T}-1\right)\right] \leq 0
$$

Note that $\left(1-\mathfrak{R}_{T} r_{T}^{p-1}\right)\left(r_{T}-1\right) \leq 0$ if and only if $\mathfrak{R}_{T}^{1 /(1-p)} \leq r_{T} \leq 1$ or $1 \leq r_{T} \leq \mathfrak{R}_{T}^{1 /(1-p)}$, hence

$$
\mathbb{E}^{\mathbb{P}^{T}}\left[\left|1-\mathfrak{R}_{T} r_{T}^{p-1}\right|\left|r_{T}-1\right|\right] \leq 2 \mathbb{E}^{\mathbb{P}^{T}}\left[\left(1-\mathfrak{R}_{T} r_{T}^{p-1}\right)\left(1-r_{T}\right) 1_{\left\{R_{T}^{1 /(1-p)} \leq r_{T} \leq 1 \text { or } 1 \leq r_{T} \leq R^{1 /(1-p)}\right\}}\right] .
$$

Let us estimate the right-hand-side expectation on $\left\{\mathfrak{R}_{T}^{1 /(1-p)} \leq r_{T} \leq 1\right\}$ and $\left\{1 \leq r_{T} \leq \mathfrak{R}_{T}^{1 /(1-p)}\right\}$ separately. On the first set, note that $\left(1-\mathfrak{R}_{T} r_{T}^{p-1}\right)\left(1-r_{T}\right) \leq\left(1-\mathfrak{R}_{T}\right)\left(1-\mathfrak{R}_{T}^{1 /(1-p)}\right)$. Then

$$
\begin{aligned}
& \mathbb{E}^{\mathbb{P}^{T}}\left[\left(1-\mathfrak{R}_{T} r_{T}^{p-1}\right)\left(1-r_{T}\right) 1_{\left\{\mathfrak{R}_{T}^{1 /(1-p)} \leq r_{T} \leq 1\right\}}\right] \\
& \leq \mathbb{E}^{\mathbb{P}^{T}}\left[\left(1-\mathfrak{R}_{T}\right)\left(1-\mathfrak{R}_{T}^{1 /(1-p)}\right) 1_{\left\{\mathfrak{R}_{T} \leq 1\right\}}\right] \\
& \leq \mathbb{P}^{T}\left(\mathfrak{R}_{T} \leq 1-\epsilon\right)+\mathbb{E}^{\mathbb{P}^{T}}\left[\left(1-\mathfrak{R}_{T}\right)\left(1-\mathfrak{R}_{T}^{1 /(1-p)}\right) 1_{\left\{1-\epsilon \leq \mathfrak{R}_{T} \leq 1\right\}}\right] \\
& \leq \mathbb{P}^{T}\left(\mathfrak{R}_{T} \leq 1-\epsilon\right)+\epsilon\left(1-(1-\epsilon)^{1 /(1-p)}\right) .
\end{aligned}
$$

Sending $T \rightarrow \infty$ then $\epsilon \downarrow 0$ and using Lemma 3.5, it follows that

$$
\lim _{T \rightarrow \infty} \mathbb{E}^{\mathbb{P}^{T}}\left[\left(1-\mathfrak{R}_{T} r_{T}^{p-1}\right)\left(1-r_{T}\right) 1_{\left\{R_{T}^{1 /(1-p)} \leq r_{T} \leq 1\right\}}\right]=0 .
$$

On the set $\left\{1 \leq r_{T} \leq \mathfrak{R}_{T}^{1 /(1-p)}\right\}$, observe that $\mathfrak{R}_{T} r_{T}^{p-1}+r_{T} \geq 2$. Then on the same set,

$$
\left(1-\mathfrak{R}_{T} r_{T}^{p-1}\right)\left(1-r_{T}\right)=\mathfrak{R}_{T} r_{T}^{p}-\mathfrak{R}_{T} r_{T}^{p-1}-r_{T}+1 \leq \mathfrak{R}_{T} r_{T}^{p}-1 .
$$

Therefore

$$
\begin{aligned}
& \mathbb{E}^{\mathbb{P}^{T}}\left[\left(1-\mathfrak{R}_{T} r_{T}^{p-1}\right)\left(1-r_{T}\right) 1_{\left\{1 \leq r_{T} \leq \mathfrak{R}_{T}^{1 /(1-p)}\right\}}\right] \\
& \leq \mathbb{E}^{\mathbb{P}^{T}}\left[\left(1-\mathfrak{R}_{T} r_{T}^{p-1}\right)\left(1-r_{T}\right) 1_{\left\{1 \leq r_{T} \leq \mathfrak{R}_{T}^{1 /(1-p)}, \mathfrak{R}_{T} \leq 1+\epsilon\right\}}\right]+\mathbb{E}^{\mathbb{P}^{T}}\left[\left(\mathfrak{R}_{T} r_{T}^{p}-1\right) 1_{\left\{1 \leq r_{T} \leq \mathfrak{R}_{T}^{1 /(1-p)}, 1+\epsilon<\mathfrak{R}_{T}\right\}}\right] \\
& =: J_{1}+J_{2} .
\end{aligned}
$$

In the previous equation, $J_{1} \leq \epsilon\left((1+\epsilon)^{1 /(1-p)}-1\right)$. Let us focus on $J_{2}$ in what follows. Since

$$
J_{2} \leq \mathbb{E}^{\mathbb{P}^{T}}\left[\left(\mathfrak{R}_{T} r_{T}^{p}-1\right) 1_{\left\{1+\epsilon<\mathfrak{R}_{T}\right\}}\right]=\mathbb{E}^{\mathbb{P}^{T}}\left[\mathfrak{R}_{T} r_{T}^{p} 1_{\left\{1+\epsilon<\mathfrak{R}_{T}\right\}}\right]-\mathbb{P}^{T}\left(1+\epsilon<\mathfrak{R}_{T}\right),
$$


and $\lim _{T \rightarrow \infty} \mathbb{P}^{T}\left(1+\epsilon<\mathfrak{R}_{T}\right)=0$ from Lemma 3.5, it suffices to estimate the first term in the previous inequality. To this end, note from (CONV) that $\left\{1+\epsilon<\mathfrak{R}_{T}\right\} \subset\left\{X_{T}^{1, T} \leq M\right\}$, for some $M$ depending on $\epsilon$. Then

$$
\begin{aligned}
\mathbb{E}^{\mathbb{P}^{T}}\left[\mathfrak{R}_{T} r_{T}^{p} 1_{\left\{1+\epsilon<\mathfrak{R}_{T}\right\}}\right] & \leq \mathbb{E}^{\mathbb{P}^{T}}\left[\mathfrak{R}_{T} r_{T}^{p} 1_{\left\{X_{T}^{1, T} \leq M\right\}}\right]=\frac{\mathbb{E}^{\mathbb{P}}\left[U^{\prime}\left(X_{T}^{1, T}\right) X_{T}^{1, T} 1_{\left\{X_{T}^{1, T} \leq M\right\}}\right]}{\mathbb{E}^{\mathbb{P}}\left[\left(X_{T}^{0, T}\right)^{p}\right]} \\
& =\frac{y^{1, T}}{y^{0, T}} \mathbb{E}^{\mathbb{P}}\left[Y_{T}^{1, T} X_{T}^{1, T} 1_{\left\{X_{T}^{1, T} \leq M\right\}}\right] .
\end{aligned}
$$

Introduce a probability measure $\mathbb{P}^{1, T}$ via

$$
\frac{d \mathbb{P}^{1, T}}{d \mathbb{P}}=Y_{T}^{1, T} X_{T}^{1, T}
$$

A line of reasoning similar to that in iii) of Remark 3.2 shows that $X^{1, T}$ has the numéraire property under $\mathbb{P}^{1, T}$. Thus, the argument in Lemma 3.3 applied to $X^{1, T}$ and $\mathbb{P}^{1, T}$ implies that $\lim _{T \rightarrow \infty} \mathbb{P}^{1, T}\left(X^{1, T} \geq M\right)=1$. The previous convergence, combined with Lemma 3.4, then implies

$$
\frac{y^{1, T}}{y^{0, T}} \mathbb{E}^{\mathbb{P}}\left[Y_{T}^{1, T} X_{T}^{1, T} 1_{\left\{X_{T}^{1, T} \leq M\right\}}\right]=\frac{y^{1, T}}{y^{0, T}} \mathbb{P}^{1, T}\left(X_{T}^{1, T} \leq M\right) \rightarrow 0, \quad \text { as } T \rightarrow \infty .
$$

Now, combining estimates on $J_{1}$ and $J_{2}$, and utilizing Lemma 3.5, we obtain from sending $T \rightarrow \infty$ then $\epsilon \downarrow 0$ that

$$
\lim _{T \rightarrow \infty} \mathbb{E}^{\mathbb{P}^{T}}\left[\left(1-\mathfrak{R}_{T} r_{T}^{p-1}\right)\left(1-r_{T}\right) 1_{\left\{1 \leq r_{T} \leq \mathfrak{R}_{T}^{1 /(1-p)}\right\}}\right]=0 .
$$

Combining the previous convergence with (3.5), the statement now follows from (3.4).

The previous result implies that $r_{T}^{T} \rightarrow 1$ under $\mathbb{P}^{T}$.

\section{Lemma 3.7. It holds that}

$$
\lim _{T \rightarrow \infty} \mathbb{P}^{T}\left(\left|r_{T}^{T}-1\right| \geq \epsilon\right)=0, \quad \text { for any } \epsilon>0 .
$$

Proof. As in the proof of Lemma 3.6, set $\mathfrak{R}_{T}=\mathfrak{R}\left(X_{T}^{1, T}\right)$ and $r_{T}=r_{T}^{T}$. Fix $\epsilon \in(0,1)$ and consider the set

$$
D^{T}=\left\{\left|r_{T}-1\right| \geq \epsilon,(1-\epsilon)^{\frac{1-p}{2}} \leq \mathfrak{R}_{T} \leq(1+\epsilon)^{\frac{1-p}{2}}\right\} .
$$

In the following, we estimate the lower bound of $\left|1-\mathfrak{R}_{T} r_{T}^{p-1}\right|$ on $D^{T}$ for the cases $r_{T} \geq 1+\epsilon$ and $r_{T} \leq 1-\epsilon$ separately.

For $r_{T} \geq 1+\epsilon$, on $D^{T}$ we have $\mathfrak{R}_{T} r_{T}^{p-1} \leq(1+\epsilon)^{\frac{p-1}{2}}<1$, whence

$$
1-\mathfrak{R}_{T} r_{T}^{p-1} \geq 1-(1+\epsilon)^{\frac{p-1}{2}}>0 ;
$$

For $r_{T} \leq 1-\epsilon$, on $D^{T}$ we have $\mathfrak{R}_{T} r_{T}^{p-1} \geq(1-\epsilon)^{\frac{p-1}{2}}>1$ on $D^{T}$ whence

$$
1-\mathfrak{R}_{T} r_{T}^{p-1} \leq 1-(1-\epsilon)^{\frac{p-1}{2}}<0 .
$$


Denote $\eta=\min \left\{1-(1+\epsilon)^{\frac{p-1}{2}},-1+(1-\epsilon)^{\frac{p-1}{2}}\right\}$. In either of the above cases, $\left|1-\mathfrak{R}_{T} r_{T}^{p-1}\right| \geq \eta$, therefore

$$
\mathbb{E}^{\mathbb{P}^{T}}\left[\left|1-\mathfrak{R}_{T} r_{T}^{p-1}\right|\left|r_{T}-1\right|\right] \geq \epsilon \eta \mathbb{P}^{T}\left(D^{T}\right) .
$$

Combining the previous inequality with Lemma 3.6 , it follows that

$$
\lim _{T \rightarrow \infty} \mathbb{P}^{T}\left(D^{T}\right)=0
$$

Now, combining the previous convergence with the second statement in Lemma 3.5, we conclude the proof.

Now we are ready to prove the main result of this subsection.

Proposition 3.8. Under Assumptions 2.1 - 2.4.

$$
\lim _{T \rightarrow \infty} \mathbb{E}^{\mathbb{P}^{T}}\left[\left|r_{T}^{T}-1\right|\right]=0
$$

Proof. As in the previous Lemmas, set $r_{T}=r_{T}^{T}$. The proof consists of the following two steps, whose combination confirms the claim. Note that for $p=0, \mathbb{P}^{T}$ below is exactly $\mathbb{P}$ and hence convergence takes place under the physical measure.

Step 1: Establish that

$$
\lim _{T \rightarrow \infty} \mathbb{E}^{\mathbb{P}^{T}}\left[\left|r_{T}-1\right| 1_{\left\{r_{T} \leq N\right\}}\right]=0, \quad \text { for any } N>2 .
$$

To this end, for any $\epsilon>0$, we have

$$
\begin{aligned}
\mathbb{E}^{\mathbb{P}^{T}}\left[\left|r_{T}-1\right| 1_{\left\{r_{T} \leq N\right\}}\right]= & \mathbb{E}^{\mathbb{P}^{T}}\left[\left|r_{T}-1\right| 1_{\left\{r_{T} \leq N,\left|r_{T}-1\right| \leq \epsilon\right\}}\right]+\mathbb{E}^{\mathbb{P}^{T}}\left[\left|r_{T}-1\right| 1_{\left\{r_{T} \leq N,\left|r_{T}-1\right|>\epsilon\right\}}\right] \\
& \leq \epsilon+(N-1) \mathbb{P}^{T}\left(\left|r_{T}-1\right|>\epsilon\right) .
\end{aligned}
$$

As $T \rightarrow \infty,(3.6)$ follows from Lemma 3.7 and the arbitrary choice of $\epsilon$.

Step 2: We also establish that

$$
\lim _{T \rightarrow \infty} \mathbb{E}^{\mathbb{P}^{T}}\left[\left|r_{T}-1\right| 1_{\left\{r_{T}>N\right\}}\right]=0, \quad \text { for any } N>2 .
$$

To this end,

$$
\mathbb{E}^{\mathbb{P}^{T}}\left[\left|r_{T}-1\right| 1_{\left\{r_{T}>N\right\}}\right] \leq \mathbb{E}^{\mathbb{P}^{T}}\left[r_{T} 1_{\left\{r_{T}>N\right\}}\right]=\mathbb{E}^{\mathbb{P}^{T}}\left[r_{T}\right]-\mathbb{E}^{\mathbb{P}^{T}}\left[\left(r_{T}-1\right) 1_{\left\{r_{T} \leq N\right\}}\right]-\mathbb{P}^{T}\left(r_{T} \leq N\right) .
$$

Note that $\mathbb{E}^{\mathbb{P}^{T}}\left[r_{T}\right] \leq 1$ due to the numéraire property of $X^{0, T}$ under $\mathbb{P}^{T}$ (cf. Remark 3.2 item iii)), $\lim _{T \rightarrow \infty} \mathbb{E}^{\mathbb{P}^{T}}\left[\left(r_{T}-1\right) 1_{\left\{r_{T} \leq N\right\}}\right]=0$ from Step 1, and $\lim _{T \rightarrow \infty} \mathbb{P}^{T}\left(r_{T} \leq N\right)=1$ from Lemma 3.7, therefore

$$
0 \leq \limsup _{T \rightarrow \infty} \mathbb{E}^{\mathbb{P}^{T}}\left[\left|r_{T}-1\right| 1_{\left\{r_{T}>N\right\}}\right] \leq 1-0-1=0
$$

which confirms (3.7). 
3.2. Convergence of Wealth Processes. The following lemma bridges this transition from the convergence of optimal payoffs to the convergence of their wealth processes.

Lemma 3.9. Consider a sequence $\left(r^{T}\right)_{T \in \mathbb{R}_{+}}$of càdlàg processes and a sequence $\left(\mathbb{P}^{T}\right)_{T \in \mathbb{R}_{+}}$of probability measures, such that:

i) For each $T \in \mathbb{R}_{+}, r^{T}$ is defined on $[0, T]$ with $r_{0}^{T}=1$ and $r_{t}^{T}>0$ for all $t \leq T, \mathbb{P}^{T}$-a.s..

ii) Each $r^{T}$ is a $\mathbb{P}^{T}$-supermartingale on $[0, T]$

iii) $\lim _{T \rightarrow \infty} \mathbb{E}^{\mathbb{P}^{T}}\left[\left|r_{T}^{T}-1\right|\right]=0$.

Then:

a) $\lim _{T \rightarrow \infty} \mathbb{P}^{T}\left(\sup _{u \in[0, T]}\left|r_{u}^{T}-1\right| \geq \epsilon\right)=0$, for any $\epsilon>0$.

b) Define $L^{T}:=\int_{0}^{\cdot}\left(1 / r_{t-}^{T}\right) d r_{t}^{T}$, i.e., $L^{T}$ is the stochastic logarithm of $r^{T}$, for each $T \in \mathbb{R}_{+}$. Then $\lim _{T \rightarrow \infty} \mathbb{P}^{T}\left(\left[L^{T}, L^{T}\right]_{T} \geq \epsilon\right)=0$, for any $\epsilon>0$, where $[\cdot, \cdot]_{T}$ is the square bracket on $[0, T]$.

Proof. This result follows from Theorem 2.5 and Remark 2.6 in Kardaras (2010). (Note that Theorem 2.5 in Kardaras (2010) is stated under a fixed probability $\overline{\mathbb{P}}$ and on a fixed time interval $[0, T]$, but its proof remains valid for a sequence of probability measures $\left(\mathbb{P}^{T}\right)_{T \in \mathbb{R}_{+}}$and a family of time intervals $\left.([0, T])_{T \in \mathbb{R}_{+}} \cdot\right)$

Combining the Lemma 3.9 with Proposition 3.8, Theorem 2.5 is proved as follows.

Proof of Theorem 2.5. The statements follow from Lemma 3.9 directly, once we check that the assumptions of Lemma 3.9 are satisfied. First, $r_{0}^{T}=1$ since both investors have the same initial capital. Second, assuming $r^{T}$ being a $\mathbb{P}^{T}$-supermartingale for a moment, then $r_{t}^{T}>0$, $\mathbb{P}^{T}$-a.s., for any $t \leq T$, because $r_{T}^{T}>0, \mathbb{P}^{T}$-a.s. (see Remark $[3.2 \mathrm{i})$ ). Third, $\lim _{T \rightarrow \infty} \mathbb{E}^{\mathbb{P}^{T}}\left[\left|r_{T}^{T}-1\right|\right]=0$ is the result of Proposition 3.8. Hence it remains to show that $r^{T}$ is a $\mathbb{P}^{T}$-supermartingale. To this end, it suffices to show that:

$$
\mathbb{E}^{\mathbb{P}^{T}}\left[X_{t} / X_{t}^{0, T} \mid \mathcal{F}_{s}\right] \leq X_{s} / X_{s}^{0, T}, \quad \text { for any } s<t \leq T \text { and } X \in \mathcal{X}^{T} .
$$

Since $X_{T}^{0, T}>0 \mathbb{P}^{T}$ a.s., Remark 3.2 i) implies that both denominators in above inequality are nonzero. To prove (3.8), fix any $A \in \mathcal{F}_{\text {s }}$, and construct the wealth process $\tilde{X} \in \mathcal{X}^{T}$ via

$$
\widetilde{X}_{u}:= \begin{cases}X_{u}^{0, T}, & u \in[0, s) \\ X_{s}^{0, T} \frac{X_{u}}{X_{s}} 1_{A}+X_{u}^{0, T} 1_{\Omega \backslash A}, & u \in[s, t) . \\ X_{s}^{0, T} \frac{X_{t}}{X_{s}} \frac{X_{u}^{0, T}}{X_{t}^{0, T}} 1_{A}+X_{u}^{0, T} 1_{\Omega \backslash A}, & u \in[t, T]\end{cases}
$$

Noting that

$$
\frac{\tilde{X}_{T}}{X_{T}^{0, T}}=\frac{X_{s}^{0, T}}{X_{s}} \frac{X_{t}}{X_{t}^{0, T}} 1_{A}+1_{\Omega \backslash A},
$$

the claim follows from $\mathbb{E}^{\mathbb{P}^{T}}\left[\widetilde{X}_{T} / X_{T}^{0, T}\right] \leq 1$ (cf. Remark 3.2 iii) ) and the arbitrary choice of $A$. 
Proof of Corollary 2.7. First, we prove that $\left(d \mathbb{P}^{T} /\left.d \mathbb{P}\right|_{\mathcal{F}_{t}}\right)_{T \geq 0}$ is a constant sequence. For any $t \leq$ $T \leq S$,

$$
\begin{aligned}
\left.\frac{d \mathbb{P}^{S}}{d \mathbb{P}}\right|_{\mathcal{F}_{t}}=\frac{\mathbb{E}_{t}^{\mathbb{P}}\left[\left(X_{S}^{0, S}\right)^{p}\right]}{\mathbb{E}^{\mathbb{P}}\left[\left(X_{S}^{0, S}\right)^{p}\right]}=\frac{\mathbb{E}_{t}^{\mathbb{P}}\left[\left(X_{T}^{0, S}\right)^{p}\left(X_{S}^{0, S} / X_{T}^{0, S}\right)^{p}\right]}{\mathbb{E}^{\mathbb{P}}\left[\left(X_{T}^{0, S}\right)^{p}\left(X_{S}^{0, S} / X_{T}^{0, S}\right)^{p}\right]}=\frac{\mathbb{E}_{t}^{\mathbb{P}}\left[\left(X_{T}^{0, S}\right)^{p}\right] \mathbb{E}_{t}^{\mathbb{P}}\left[\left(X_{S}^{0, S} / X_{T}^{0, S}\right)^{p}\right]}{\mathbb{E}^{\mathbb{P}}\left[\left(X_{T}^{0, S}\right)^{p}\right] \mathbb{E}^{\mathbb{P}}\left[\left(X_{S}^{0, S} / X_{T}^{0, S}\right)^{p}\right]} \\
=\frac{\mathbb{E}_{t}^{\mathbb{P}}\left[\left(X_{T}^{0, S}\right)^{p}\right]}{\mathbb{E}^{\mathbb{P}}\left[\left(X_{T}^{0, S}\right)^{p}\right]}=\frac{\mathbb{E}_{t}^{\mathbb{P}}\left[\left(X_{T}^{0, T}\right)^{p}\right]}{\mathbb{E}^{\mathbb{P}}\left[\left(X_{T}^{0, T}\right)^{p}\right]}=\left.\frac{d \mathbb{P}^{T}}{d \mathbb{P}}\right|_{\mathcal{F}_{t}} .
\end{aligned}
$$

Here, the third equality follows from the assumption that $X_{T}^{0, S}$ and $X_{S}^{0, S} / X_{T}^{0, S}$ are independent; the fourth equality holds since $X_{S}^{0, S} / X_{T}^{0, S}$ is independent of $\mathcal{F}_{t}$; and the fifth equality holds by the myopic optimality $X_{T}^{0, T}=X_{T}^{0, S}$.

Second, note that $d \mathbb{P}^{T} /\left.d \mathbb{P}\right|_{\mathcal{F}}$ is a strictly positive martingale; see the discussion after (2.4), it then induces a probability measure $\tilde{\mathbb{P}}$, which is equivalent to $\mathbb{P}$ on $\mathcal{F}_{t}$. As a result, the rest statements follows from Theorem 2.5 and Remark 2.6 iii) directly.

\section{Proof of the Turnpike for Diffusions}

This section contains the proofs of the statements in section 2.2, and is broken into four subsections. The first one details properties of the measures $\left(\hat{\mathbb{P}}^{\xi}\right)_{\xi \in \mathbb{R}^{d} \times E}$. The second subsection constructs the reduced value function $v^{T}$ in (2.21). The third section establishes the precise relations between the conditional densities $d \mathbb{P}^{T, y} /\left.d \mathbb{P}^{y}\right|_{\mathcal{F}_{t}}, d \hat{\mathbb{P}}^{y} /\left.d \mathbb{P}^{y}\right|_{\mathcal{F}_{t}}$ and the wealth processes $X^{0, T} / \hat{X}$. The last subsection contains the proofs of the main results.

Remark 4.1. To ease notation, denote in the sequel:

$$
\mathcal{E}\left(\int H d W\right)_{t, s}:=\exp \left(\int_{t}^{s} H_{u} d W_{u}-\frac{1}{2} \int_{t}^{s}\left\|H_{u}\right\|^{2} d u\right) \quad \text { for any integrand } H \text { and } t \leq s,
$$

and by $\mathcal{E}\left(\int H d W\right)_{t}=\mathcal{E}\left(\int H d W\right)_{0, t}$.

4.1. The long run measure $\hat{\mathbb{P}}^{\xi}$. Recall the following terminology from ergodic theory for diffusions (see Pinsky (1995) for a more thorough treatment). Let $L$ be as in (2.2). Suppose the martingale problem for $L$ is well posed on $D$, and denote its solution by $\left(\mathbb{P}^{x}\right)_{x \in D}$, with coordinate process $\Xi$. Denote by $L^{*}$ the formal adjoint to $L$. Note that under the given regularity conditions $L^{*}$ is a second order differential operator as well.

$\Xi$ is recurrent under $\left(\mathbb{P}^{x}\right)_{x \in D}$ if $\mathbb{P}^{x}(\tau(\epsilon, y)<\infty)=1$ for any $(x, y) \in D^{2}$ and $\epsilon>0$, where $\tau(\epsilon, y)=\inf \left\{t \geq 0|| \Xi_{t}-y \mid \leq \epsilon\right\}$. If $\Xi$ is recurrent then it is positive recurrent, or ergodic if there exists a strictly positive $\varphi^{*} \in C^{2, \gamma}\left(D, \mathbb{R}^{k}\right)$ such that $L^{*} \varphi^{*}=0$ and $\int_{D} \varphi^{*}(y) d y<\infty$. If $\Xi$ is recurrent, but not positive recurrent, it is null recurrent. 
Lemma 4.2. Let Assumptions 2.8 and 2.14 hold. Let $\mathcal{L}$ be as in (2.13) and define $\mathcal{L}^{\hat{v}, 0}$ by

$$
\mathcal{L}^{\hat{v}, 0}=\mathcal{L}+A \frac{\hat{v}_{y}}{\hat{v}} \partial_{y} .
$$

Then, there exists a unique solution $\left(\hat{\mathbb{P}}_{Y}^{y}\right)_{y \in E}$ to the martingale problem for $\mathcal{L}^{\hat{v}, 0}$ on E. Furthermore, the coordinate mapping process $Y$ is positive recurrent under $\left(\hat{\mathbb{P}}_{Y}^{y}\right)_{y \in E}$ with invariant density $\mu(y)=$ $\hat{v}^{2}(y) \hat{m}(y)$, where $\hat{m}$ is defined in (2.16). Therefore, for all functions $f \in L^{1}(E, \mu)$ and all $t>0$ :

$$
\lim _{T \uparrow \infty} \mathbb{E}^{\hat{\mathbb{P}}_{Y}^{y}}\left[f\left(Y_{T-t}\right)\right]=\int_{E} f(y) \hat{v}^{2}(y) \hat{m}(y) d y .
$$

Proof. Since (2.14) and (2.15) hols, Pinsky (1995, Theorem 5.1.10, Corollary 5.1.11) implies that: a) $\left(\hat{\mathbb{P}}_{Y}^{y}\right)_{y \in E}$ exists and is unique, b) $Y$ is positive recurrent under $\left(\hat{\mathbb{P}}_{Y}^{y}\right)_{u \in E}$, and c) $Y$ has invariant density $\hat{v}^{2}(y) \hat{m}(y)$. That (4.2) holds for $f \in L^{1}(E, \mu)$ follows from Pinchover (1992, Theorem 1.2 (iii), Eqns (3.29) and (3.30)).

Lemma 4.3. Let Assumptions 2.8 and 2.14 hold. Then:

i) There exists a unique solution $\left(\hat{\mathbb{P}}^{\xi}\right)_{\xi \in \mathbb{R}^{d} \times E}$ to the martingale problem for $\hat{\mathcal{L}}$ on $\mathbb{R}^{d} \times E$ where $\hat{\mathcal{L}}$ is given in (2.17).

ii) $\hat{\mathbb{P}}^{\xi} \sim \mathbb{P}^{\xi}$ for any $\xi \in \mathbb{R}^{d} \times E$.

Proof. For any integer $n$ denote by $\Omega^{n}$ be the space of continuous maps $\omega: \mathbb{R}_{+} \rightarrow \mathbb{R}^{n}$ and $\mathcal{B}^{n}$ be the sigma algebra generated by the coordinate process $\Xi$ defined by $\Xi_{t}(\omega)=\omega_{t}$ for $\omega \in \Omega^{n}$. By Lemma 4.2, there is a unique solution $\left(\hat{\mathbb{P}}_{Y}^{y}\right)_{y \in E} \in M_{1}\left(\Omega^{1}, \mathcal{B}^{1}\right)$ to the martingale problem on $E$ for the operator $\mathcal{L}^{\hat{v}, 0}$ given in (4.1). Set $\Omega=\Omega^{d+1}, \mathcal{F}=\mathcal{B}^{d+1}$ and $\mathcal{F}_{t}=\mathcal{B}_{t+}^{d+1}, t \geq 0$. Let $W^{d}$ denote d-dimensional Wiener measure on the first $d$ coordinates (along with the associated sigma algebra) and set $B=\left(\Xi^{1}, \ldots, \Xi^{d}\right), Y=\Xi^{d+1}$. For any $z \in \mathbb{R}^{d}$ define the processes $X, W$ by

$$
X_{t}:=z-q \int_{0}^{t} \bar{\rho}^{\prime} \sigma^{\prime} \Sigma^{-1}\left(\mu+\delta \Upsilon \frac{\hat{v}_{y}}{\hat{v}}\right)\left(Y_{s}\right) d s+B_{t} ; \quad W_{t}=\int_{0}^{t} a^{-1}\left(Y_{s}\right)\left(d Y_{s}-b\left(Y_{s}\right) d s\right) .
$$

Clearly for $\xi=(z, y)$ it follows that, $\left((X, Y),(B, W),\left(\Omega, \mathcal{F},\left(\mathcal{F}_{t}\right)_{t \geq 0}, W^{d} \times \hat{\mathbb{P}}_{Y}^{y}\right)\right)$ is a weak solution to the SDE associated to the operator $\hat{\mathcal{L}}$.

Since weak solutions induce solutions to the martingale problem via Ito's formula, it follows that if $\hat{\mathbb{P}}^{\xi} \in M_{1}(\Omega, \mathcal{F})$ is defined by $\hat{\mathbb{P}}^{\xi}(A)=W^{d} \times \hat{\mathbb{P}}^{y}((X, Y) \in A)$ with $A \in \mathcal{F}$, then $\left(\hat{\mathbb{P}}^{\xi}\right)_{\xi \in \mathbb{R}^{d} \times E}$ solves the martingale problem for $\hat{\mathcal{L}}$.

Part (ii) follows from (Cheridito et al., 2005, Remark 2.6) and Lemma 2.11, Note that the assumption in Cheridito et al. (2005) is satisfied in view of Assumption 2.8, $\hat{v}>0$ and $\hat{v} \in C^{2}(E)$ in Assumption 2.14. 
Remark 4.4. As in the proof of Lemma 4.3, for all $\xi=(z, y)$ with $z \in \mathbb{R}^{d}$ and $y \in E$, if $Y$ denotes the $(d+1)^{s t}$ coordinate, then

$$
\hat{\mathbb{P}}^{\xi}(Y \in A)=\hat{\mathbb{P}}_{Y}^{y}(Y \in A) ; \quad A \in \mathcal{B}^{1} .
$$

Thus, since $Y$ is positive recurrent under $\left(\hat{\mathbb{P}}_{Y}^{y}\right)_{y \in E}$ by Lemma 4.2, $Y$ is positive recurrent under $\left(\hat{\mathbb{P}}^{\xi}\right)_{\xi \in \mathbb{R}^{d} \times E}$ with the same invariant density $\mu$ as in Lemma 4.2. Therefore, the ergodic result in (4.2) applies to $\hat{\mathbb{P}}^{\xi}$ for any $\xi \in \mathbb{R}^{d} \times E$.

4.2. Construction of $v^{T}$. The solution $v^{T}(t, y)$ to (2.21) is constructed from the long-run solution $\hat{v}(y)$ of Assumption 2.14, Recall that $\hat{\mathbb{P}}^{\xi}$ is denoted by $\hat{\mathbb{P}}^{y}$ for $\xi=(0, y)$. Now, consider the function $h^{T}$ defined as:

$$
h^{T}(t, y):=\mathbb{E}^{\hat{\mathbb{P}}^{y}}\left[\frac{1}{\hat{v}\left(Y_{T-t}\right)}\right], \quad(t, y) \in[0, T] \times E .
$$

The candidate reduced value function is

$$
v^{T}(t, y):=e^{\lambda_{c}(T-t)} \hat{v}(y) h^{T}(t, y) .
$$

Thus, $h^{T}$ is the ratio between $v^{T}$ and its long-run analogue $e^{\lambda_{c}(T-\cdot)} \hat{v}$. The verification result Proposition 4.7 below confirms that $v^{T}$ is a strictly positive classical solution to (2.21) and the relation $u^{T}(t, x, y)=\left(x^{p} / p\right)\left(v^{T}(t, y)\right)^{\delta}$ holds for $(t, x, y) \in[0, T] \times \mathbb{R}_{+} \times E$.

As a first step to proving Proposition 4.7, the next result characterizes the function $h^{T}$. Clearly, $h^{T}(t, y)>0$ for $(t, y) \in[0, T] \times E$.

Proposition 4.5. Let Assumptions 2.8, 2.9, 2.13 and 2.14 hold. Then $h^{T}(t, y)<\infty$ for all $(t, y) \in[0, T] \times E, h^{T}(t, y) \in C^{1,2}((0, T) \times E)$, and $h^{T}$ satisfies

$$
\begin{aligned}
& \partial_{t} h^{T}+\mathcal{L}^{\hat{v}, 0} h^{T}=0, \quad(t, y) \in(0, T) \times E \\
& h^{T}(T, y)=\frac{1}{\hat{v}(y)}, \quad y \in E
\end{aligned}
$$

where $\mathcal{L}^{\hat{v}, 0}$ is defined in (4.1). Furthermore, the process

$$
\frac{h^{T}\left(t, Y_{t}\right)}{h^{T}(0, y)} ; \quad 0 \leq t \leq T
$$

is a $\hat{\mathbb{P}}^{y}$ martingale on $[0, T]$ with constant expectation 1 . Lastly, for all $t>0$ and $y \in E$ it follows $\hat{\mathbb{P}}^{y}$ almost surely that

$$
\lim _{T \rightarrow \infty} \frac{h^{T}\left(t, Y_{t}\right)}{h^{T}(0, y)}=1
$$

Proof. The proof consists of several steps.

Step 1: $h^{T}(t, y)<\infty$ for all $(t, y) \in[0, T] \times E$. Note that (2.12) implies

$$
\mathcal{L}^{\hat{v}, 0}\left(\frac{1}{\hat{v}}\right)=-\frac{\mathcal{L} \hat{v}}{\hat{v}^{2}}=\frac{c-\lambda_{c}}{\hat{v}} .
$$


Combining (4.8) with $\sup _{y \in E} c(y)<\infty$ in Assumption 2.13, there exists some $K>0$ such that

$$
\left(\partial_{t}+\mathcal{L}^{\hat{v}, 0}\right)\left(\frac{e^{-K t}}{\hat{v}(y)}\right) \leq 0
$$

Thus, using the strict positivity of $\hat{v}$, Assumption 2.8 and Fatou's lemma, it follows that:

$$
h^{T}(t, y)=\mathbb{E}^{\hat{\mathbb{P}}^{y}}\left[\left(\hat{v}\left(Y_{T-t}\right)\right)^{-1}\right] \leq \frac{e^{K(T-t)}}{\hat{v}(y)}<\infty .
$$

Step 2: $h^{T} \in C^{1,2}((0, T) \times E)$ satisfies (4.5). To this end, the classical version of the FeynmanKac formula (see Theorem 5.3 in Friedman (1975) pp. 148) does not apply directly because a) the operator $\mathcal{L}^{\hat{v}, 0}$ is not assumed to be uniformly elliptic on $E$, and b) $(\hat{v})^{-1}$ may grow faster than polynomial near the boundary of $E$. Rather, the statement follows from Theorem 1 in Heath and Schweizer (2000), which yields that $h^{T}$ is a classical solution of (4.5).

To check that the assumptions of Theorem 1 in Heath and Schweizer (2000) are satisfied, note that, since $A$ is locally Lipschitz on $E$ due to Assumption 2.8, Lemma 1.1 in Friedman (1975) pp. 128 implies that $a$ is also locally Lipschitz on $E$. On the other hand, the local Lipschitz continuity of $B+A \hat{v}_{y} / \hat{v}$ is ensured by Assumption 2.8 and $\hat{v} \in C^{2}(E)$. Hence (A1) in Heath and Schweizer (2000) is satisfied. Second, (A2) in Heath and Schweizer (2000) holds thanks to the well-posedness of the martingale problem for $\hat{\mathcal{L}}$ on $\mathbb{R}^{d} \times E$, as proved in Lemma 4.3. Third, (A3') in Heath and Schweizer (2000), (A3a')-(A3d') are clearly satisfied under our assumptions.

In order to check (A3e'), it suffices to show that $h^{T}$ is continuous in any compact sub-domain of $(0, T) \times E$. To this end, recall that the domain is $E=(\alpha, \beta)$ for $-\infty \leq \alpha<\beta \leq \infty$. Let $\left\{\alpha_{m}\right\}$ and $\left\{\beta_{m}\right\}$ two sequences such that $\alpha_{m}<\beta_{m}$ for all $m, \alpha_{m}$ strictly decreases to $\alpha$, and $\beta_{m}$ strictly increases to $\beta$. Set $E_{m}=\left(\alpha_{m}, \beta_{m}\right)$. For each $m$ there exists a function $\psi_{m}(y) \in C^{\infty}(E)$ such that a) $\psi_{m}(y) \leq 1$, b) $\psi_{m}(y)=1$ on $E_{m}$, and c) $\psi_{m}(y)=0$ on $E \cap E_{m+1}^{c}$. To construct such $\psi_{m}$ let $\varepsilon_{m}=\frac{1}{3} \min \left\{\beta_{m+1}-\beta_{m}, \alpha_{m}-\alpha_{m+1}\right\}$ and then take

$$
\psi_{m}(y)=\eta_{\varepsilon_{m}} * 1_{\left\{\alpha_{m}-\varepsilon_{m}, \beta_{m}+\varepsilon_{m}\right\}}(y),
$$

where $\eta_{\varepsilon_{m}}$ is the standard mollifier and $*$ is the convolution operator. Define the functions $f_{m}$ and $h^{T, m}$ by

$$
f_{m}(y)=\frac{\psi_{m}(y)}{\hat{v}(y)} \quad \text { and } \quad h^{T, m}(t, y)=\mathbb{E}^{\hat{P}^{y}}\left[f_{m}\left(Y_{T-t}\right)\right]
$$

By construction, for all $y \in E, \uparrow \lim _{m \uparrow \infty} f_{m}(y)=(\hat{v}(y))^{-1}$. It then follows from the monotone convergence theorem and (4.9) that $\lim _{m \uparrow \infty} h^{T, m}(t, y)=h^{T}(t, y)$.

Since $\hat{v} \in C^{2}(E)$ and $\hat{v}>0$, each $f_{m}(y) \in C^{2}(E)$ is bounded. It then follows from the Feller property for $\hat{\mathbb{P}}^{y}$ (see Theorem 1.13.1 in Pinsky (1995)) that $h^{T, m}$ is continuous in $y$. On the other hand, by construction of $f_{m}$ and (4.8), there exists a constant $K_{m}>0$ such that

$$
a\left|\dot{f}_{m}\right| \leq K_{m}, \quad\left|\mathcal{L}^{\hat{v}, 0} f_{m}\right| \leq K_{m}, \quad \text { on } E .
$$


Moreover, Ito's formula gives that, for any $0 \leq s \leq t \leq T$,

$$
f_{m}\left(Y_{t}\right)=f_{m}\left(Y_{s}\right)+\int_{s}^{t} \mathcal{L}^{\hat{v}, 0} f_{m}\left(Y_{u}\right) d u+\int_{s}^{t} a \dot{f}_{n}\left(Y_{u}\right) d \hat{W}_{u}
$$

Combining the previous equation with estimates in (4.10), it follows that:

$$
\sup _{y \in E}\left|\mathbb{E}^{\hat{\mathbb{P}}^{y}}\left[f_{m}\left(Y_{t}\right)-f_{m}\left(Y_{s}\right)\right]\right| \leq K_{m}(t-s) .
$$

Therefore, $h^{T, m}$ is uniformly continuous in $t$. Combining with the continuity of $h^{T, m}$ in $y$, we conclude that $h^{T, m}$ is jointly continuous in $(t, y)$ on $[0, T] \times E$.

Note that the operator $\mathcal{L}^{\hat{v}, 0}$ is uniformly elliptic in the parabolic domain $(0, T) \times E_{m}$. It then follows from a straightforward calculation that $h^{T, m}$ satisfies the differential equation:

$$
\partial_{t} h^{T, m}+\mathcal{L}^{\hat{v}, 0} h^{T, m}=0 \quad(t, y) \in(0, T) \times E_{m} .
$$

Note that $\left(h^{T, m}\right)_{m \geq 0}$ is uniformly bounded from above by $h^{T}$, which is finite on $[0, T] \times E_{m}$. Appealing to the interior Schauder estimate (see e.g. Theorem 15 in Friedman (1964) pp. 80), there exists a subsequence $\left(h^{T, m^{\prime}}\right)_{m^{\prime}}$ which converges to $h^{T}$ uniformly in $(0, T) \times D$ for any compact subdomain $D$ of $E_{m}$. Since each $h^{T, m^{\prime}}$ is continuous and the convergence is uniform, we confirm that $h^{T}$ is continuous in $(0, T) \times D$. Since the choice of $D$ is arbitrary in $E_{m},($ A3e') in Heath and Schweizer (2000) is satisfied. This proves that $h^{T} \in C^{1,2}((0, T) \times E)$ and satisfies (4.5).

Step 3: Remaining statements. By definition of the martingale problem, the process in (4.6) is a non-negative local martingale, and hence a super-martingale. Furthermore, for $y \in E$, by construction of $h^{T}$

$$
\mathbb{E}^{\hat{\mathbb{P}}^{y}}\left[\frac{h^{T}\left(T, Y_{T}\right)}{h^{T}(0, y)}\right]=\frac{1}{\mathbb{E}^{\hat{P}^{y}}\left[\hat{v}\left(Y_{T}\right)^{-1}\right]} \mathbb{E}^{\hat{\mathbb{P}}^{y}}\left[\hat{v}\left(Y_{T}\right)^{-1}\right]=1,
$$

proving the martingale property on $[0, T]$. Lastly, (4.7) follows from (4.2) in Lemma 4.2 and Remark 4.4, since (2.15) in Assumption 2.14 implies that $(\hat{v})^{-1} \in L^{1}\left(E, \hat{v}^{2} \hat{m}\right)$. Thus, for all $t>0, y \in E$,

$$
\lim _{T \rightarrow \infty} h^{T}(t, y)=\int_{E} \hat{v}(z) \hat{m}(z) d z .
$$

which gives the result by taking $y=Y_{t}$ for a fixed $t$.

The next step towards the verification result in Proposition 4.7 is to connect solutions $v^{T}$ to the PDE in (2.21) to the value function $u^{T}$ of (2.11). This task requires additional notation.

Let Assumption 2.8 and 2.9 hold. For any strictly positive $w(t, y) \in C^{1,2}((0, T) \times E)$, define the process

$$
D_{t}^{w}:=\mathcal{E}\left(\int\left(-q \Upsilon^{\prime} \Sigma^{-1} \mu+A \frac{w_{y}}{w}\right)^{\prime} \frac{1}{a} d W-q \int\left(\Sigma^{-1} \mu+\Sigma^{-1} \Upsilon \delta \frac{w_{y}}{w}\right)^{\prime} \sigma \bar{\rho} d B\right)_{t},
$$


Here, $(B, W)$ is a $(d+1)$-dimensional $\mathbb{P}^{y}$ Brownian Motion. $B$ is formed by the first $d$ coordinates of $\Xi$ and $W$ is from Remark 2.12. Note that $B$ is $\mathbb{P}^{y}$ independent of $Y$. For $t \leq s \leq T$ define $D_{t, s}^{w}$ as in Remark 4.1. Denote the portfolio

$$
\pi^{w}=\frac{1}{1-p} \Sigma^{-1}\left(\mu+\delta \Upsilon \frac{w_{y}}{w}\right)
$$

evaluated at $\left(t, Y_{t}\right)$, and by $X^{\pi, w}$ the corresponding wealth process. Set $\eta^{w}=\delta w_{y} / w$ (also evaluated at $\left.\left(t, Y_{t}\right)\right)$, and define the process $M^{\eta, w}$ via

$$
M_{t}^{\eta, w}=e^{-\int_{0}^{t} r d \tau} \mathcal{E}\left(\int\left(-\Upsilon^{\prime} \Sigma^{-1} \mu+\left(A-\Upsilon^{\prime} \Sigma^{-1} \Upsilon\right) \eta^{w}\right)^{\prime} \frac{1}{a} d W-\int\left(\Sigma^{-1} \mu+\Sigma^{-1} \Upsilon \eta^{w}\right)^{\prime} \sigma \bar{\rho} d B\right)_{t} .
$$

The following proposition is crucial to check the optimality of both finite horizon and long-run optimal portfolios, and to compare their terminal wealths. A similar statement for the long-run limit is in (Guasoni and Robertson, 2009, Theorem 7).

Proposition 4.6. Let Assumptions 2.8 and 2.9 hold. Assume there exists a function $w:[0, T] \times$ $E \rightarrow \mathbb{R}$ and a constant $\lambda \in \mathbb{R}$, such that $w \in C^{1,2}((0, T) \times E, \mathbb{R})$ is strictly positive and satisfies the differential expression

$$
\partial_{t} w+\mathcal{L} w+(c-\lambda) w=0, \quad(t, y) \in(0, T) \times E .
$$

Then the following conclusions hold:

(i) For all admissible portfolios $\pi$ and all $y \in E$, the process $X^{\pi} M^{\eta, w}$ is a non-negative supermartingale under $\mathbb{P}^{y}$ where $M^{\eta, w}$ is given in (4.13).

(ii) For all $t \leq s \leq T$ and $y \in E$ the processes $X^{\pi, w}$ and $M^{\eta, w}$ satisfy the $\mathbb{P}^{y}$ almost sure identities

$$
\begin{aligned}
& \left(X_{s}^{\pi, w}\right)^{p}=\left(X_{t}^{\pi, w}\right)^{p}\left(w\left(t, Y_{t}\right) e^{\lambda(s-t)}\right)^{\delta} D_{t, s}^{w} w\left(s, Y_{s}\right)^{-\delta} \\
& \left(M_{s}^{\eta, w}\right)^{q}=\left(M_{t}^{\eta, w}\right)^{q}\left(w\left(t, Y_{t}\right) e^{\lambda(s-t)}\right)^{\frac{\delta}{1-p}} D_{t, s}^{w} w\left(s, Y_{s}\right)^{-\frac{\delta}{1-p}},
\end{aligned}
$$

where $\pi^{w}, M^{\eta, w}$ and $D^{w}$ are as in (4.12), (4.13) and (4.11) respectively.

Proof. Given $w$, it is clear, using stochastic integration by parts, that for $i=1, \ldots, d$ the process $M^{\eta, w} S^{i}$ is a non-negative supermartingale under $\mathbb{P}^{y}$ for any $y \in E$. Thus, part $(i)$ follows.

It remains the show the almost-sure identities. To this end fix $t \leq s \leq T$. By (2.10) it follows that

$$
\begin{aligned}
& \frac{\left(X_{s}^{\pi, w}\right)^{p}}{\left(X_{t}^{\pi, w}\right)^{p}}=\exp \left(\int_{t}^{s}\left(p \mu^{\prime} \pi^{w}+p r-\frac{p}{2}\left(\pi^{w}\right)^{\prime} \Sigma \pi^{w}\right) d \tau+p \int_{t}^{s}\left(\pi^{w}\right)^{\prime} \sigma d Z_{\tau}\right) \\
& \frac{\left(M_{s}^{\eta, w}\right)^{q}}{\left(M_{t}^{\eta, w}\right)^{q}}=e^{-q \int_{t}^{s} r d \tau} \mathcal{E}\left(\int\left(-\Upsilon^{\prime} \Sigma^{-1} \mu+\left(A-\Upsilon^{\prime} \Sigma^{-1} \Upsilon\right) \eta^{w}\right)^{\prime} \frac{1}{a} d W-\int\left(\Sigma^{-1} \mu+\Sigma^{-1} \Upsilon \eta^{w}\right)^{\prime} \sigma \bar{\rho} d B\right)_{t, s}^{q}
\end{aligned}
$$


Taking logarithms and expanding $Z=\rho W+\bar{\rho} B$, the first equality is equivalent to

$$
\begin{aligned}
\int_{t}^{s}\left(p \mu^{\prime} \pi^{w}+p r-\frac{p}{2}\left(\pi^{w}\right)^{\prime} \Sigma \pi^{w}\right) d \tau & +p \int_{t}^{s}\left(\pi^{w}\right)^{\prime} \sigma \rho d W_{\tau}+p \int_{t}^{s}\left(\pi^{w}\right)^{\prime} \sigma \bar{\rho} d B_{\tau} \\
& =\delta \log w\left(t, Y_{t}\right)+\delta \lambda(s-t)+\log D_{t, s}^{w}-\delta \log w\left(s, Y_{s}\right) .
\end{aligned}
$$

Multidimensional notation makes calculations more transparent. Set $\omega:=\delta \log w$. Then $\omega$ solves the quasi-linear differential expression

$$
\partial_{t} \omega+\mathcal{L} \omega+\frac{1}{2} \nabla \omega^{\prime}\left(A-q \Upsilon^{\prime} \Sigma^{-1} \Upsilon\right) \nabla \omega+\delta(c-\lambda)=0, \quad(t, y) \in(0, T) \times E,
$$

because $\delta^{-1} A=A-q \Upsilon^{\prime} \Sigma^{-1} \Upsilon$. Since $\mathcal{L}=(1 / 2) A \partial_{y y}+\left(b-q \Upsilon^{\prime} \Sigma^{-1} \mu\right) \partial_{y}$, Ito's formula implies that under $\mathbb{P}^{y}$, after expanding $c=(1 / \delta)\left(p r-(1 / 2) q \mu^{\prime} \Sigma^{-1} \mu\right)$,

$$
\int_{t}^{s}\left(q \mu^{\prime} \Sigma^{-1} \Upsilon \nabla \omega-\frac{1}{2} \nabla \omega^{\prime}\left(A-q \Upsilon^{\prime} \Sigma^{-1} \Upsilon\right) \nabla \omega+\delta \lambda-\left(p r-\frac{1}{2} q \mu^{\prime} \Sigma^{-1} \mu\right)\right) d \tau+\int_{t}^{s} \nabla \omega^{\prime} a d W_{\tau} .
$$

Again, using $\delta^{-1} A=A-q \Upsilon^{\prime} \Sigma^{-1} \Upsilon$ and noting that $\delta w_{y} / w=\omega_{y}$, after some simplifications it follows that:

$$
\begin{aligned}
\log D_{t, s}^{w} & =\int_{t}^{s}\left(-q \mu^{\prime} \Sigma^{-1} \Upsilon+\nabla \omega^{\prime}\left(A-q \Upsilon^{\prime} \Sigma^{-1} \Upsilon\right)\right) \frac{1}{a} d W_{\tau} \\
& -q \int_{t}^{s}\left(\mu^{\prime} \Sigma^{-1}+\nabla \omega \Upsilon^{\prime} \Sigma^{-1}\right) \sigma \bar{\rho} d B_{\tau} \\
& +\int_{t}^{s}\left(-\frac{1}{2} q^{2} \mu^{\prime} \Sigma^{-1} \mu+q(1-q) \mu^{\prime} \Sigma^{-1} \Upsilon \nabla \omega-\frac{1}{2} \nabla \omega^{\prime}\left(A-\left(2 q-q^{2}\right) \Upsilon^{\prime} \Sigma^{-1} \Upsilon\right) \nabla \omega\right) d \tau
\end{aligned}
$$

Lastly, plugging in for $\pi^{w}$ yields

$$
\begin{aligned}
& \int_{t}^{s}\left(p \mu^{\prime} \pi^{w}+p r-\frac{p}{2}\left(\pi^{w}\right)^{\prime} \Sigma \pi^{w}\right) d \tau+p \int_{t}^{s}\left(\pi^{w}\right)^{\prime} \sigma \rho d W_{\tau}+p \int_{t}^{s}\left(\pi^{w}\right)^{\prime} \sigma \bar{\rho} d B_{\tau} \\
& =\int_{t}^{s}\left(p r-\frac{1}{2} q(1+q) \mu^{\prime} \Sigma^{-1} \mu-q^{2} \mu^{\prime} \Sigma^{-1} \Upsilon \nabla \omega-\frac{1}{2} q(q-1) \nabla \omega^{\prime} \Upsilon^{\prime} \Sigma^{-1} \Upsilon \nabla \omega\right) d \tau \\
& \quad-q \int_{t}^{s}\left(\mu^{\prime}+\nabla \omega^{\prime} \Upsilon^{\prime}\right) \Sigma^{-1} \sigma \rho d W_{\tau}-q \int_{t}^{s}\left(\mu^{\prime}+\nabla \omega^{\prime} \Upsilon^{\prime}\right) \Sigma^{-1} \sigma \bar{\rho} d B_{\tau}
\end{aligned}
$$

Now, using (4.17), (4.18) and (4.19), the equality in (4.16) follows by matching the respective $d \tau$, $d W$ and $d B$ terms. 
The proof for the second identity in (4.14) is similar. Given (4.15), it suffices to show that, by taking logarithms

$$
\begin{array}{r}
-q \int_{t}^{s} r d \tau+q \log \mathcal{E}\left(\int\left(-\Upsilon^{\prime} \Sigma^{-1} \mu+\left(A-\Upsilon^{\prime} \Sigma^{-1} \Upsilon\right) \eta^{w}\right) \frac{1}{a} d W-\int\left(\Sigma^{-1} \mu+\Sigma^{-1} \Upsilon \eta^{w}\right) \sigma \bar{\rho} d B\right)_{t, s} \\
=\frac{\delta}{1-p} \log w\left(t, Y_{t}\right)+\frac{\delta}{1-p} \lambda(s-t)+\log D_{t, s}^{w}-\frac{\delta}{1-p} \log w\left(s, Y_{s}\right) .
\end{array}
$$

The equality in (4.17) (multiplied by $1 /(1-p)$ ), combined with that in (4.18) yield an expression for the right hand side of the above equation in terms of integrals from $s$ to $t$ of $d \tau, d W$ and $d B$. As for the left hand side, a lengthy calculation shows that

$$
\begin{aligned}
& -q \int_{t}^{s} r d \tau+q \log \mathcal{E}\left(\int\left(-\Upsilon^{\prime} \Sigma^{-1} \mu+\left(A-\Upsilon^{\prime} \Sigma^{-1} \Upsilon\right) \eta^{w}\right)^{\prime} \frac{1}{a} d W-\int\left(\Sigma^{-1} \mu+\Sigma^{-1} \Upsilon \eta^{w}\right)^{\prime} \sigma \bar{\rho} d B\right)_{t, s} \\
= & \int_{t}^{s}\left(-q r-\frac{1}{2} q \mu^{\prime} \Sigma^{-1} \mu-\frac{1}{2} q \nabla \omega^{\prime}\left(A-\Upsilon^{\prime} \Sigma^{-1} \Upsilon\right) \nabla \omega\right) d \tau \\
& +q \int_{t}^{s}\left(-\mu^{\prime} \Sigma^{-1} \Upsilon+\nabla \omega^{\prime}\left(A-\Upsilon^{\prime} \Sigma^{-1} \Upsilon\right)\right) \frac{1}{a} d W_{\tau} \\
& -q \int_{t}^{2}\left(\mu^{\prime} \Sigma^{-1}+\nabla \omega^{\prime} \Upsilon^{\prime} \Sigma^{-1}\right) \sigma \bar{\rho} d B_{\tau} .
\end{aligned}
$$

Thus, using (4.17), (4.18) and (4.21), the equality in (4.20) follows by matching $d \tau, d W$ and $d B$ terms.

The verification result for the finite horizon problem now follows.

Proposition 4.7. Let Assumptions 2.8, 2.9, 2.13, and 2.14 hold. Define $v^{T}$ by (4.4). Then:

(i) $v^{T}>0, v^{T} \in C^{1,2}((0, T) \times E)$, and it solves (2.21).

(ii) $u^{T}(t, x, y)=\frac{x^{p}}{p}\left(v^{T}(t, y)\right)^{\delta}$ on $[0, T] \times \mathbb{R}_{+} \times E$ and $\pi^{T}$ in (2.22) is the optimal portfolio.

Proof. Clearly, the positivity of $h^{T}$ and $\hat{v}$ yield that of $v^{T}$. Furthermore, given that $h^{T}$ solves (4.5), long but straightforward calculations using (2.12) show that $v^{T}$ solves (2.21). Moreover, $v^{T} \in C^{1,2}((0, T) \times E)$ because $\hat{v} \in C^{2}(E)$ and $h^{T} \in C^{1,2}((0, T) \times E)$. This proves $(i)$.

As for part $i$ ), by applying Proposition 4.6 to $w=v^{T}, \lambda=0$ it follows by evaluating (4.14) at $t=t, s=T$ that for the portfolio in (2.22) and the process $M^{\eta, v^{T}}$ from (4.13) (recall the definition of $D^{w}$ given in (4.11))

$$
\mathbb{E}^{\mathbb{P}^{\xi, t}}\left[\frac{1}{p}\left(X_{T}^{\pi^{T}}\right)^{p}\right]=\frac{x^{p}}{p}\left(v^{T}(t, y)\right)^{\delta} \mathbb{E}^{\mathbb{P}^{\xi, t}}\left[D_{t, T}^{v^{T}}\right]
$$

since $v^{T}(T, y)=1$. In a similar manner

$$
\frac{x^{p}}{p} \mathbb{E}^{\mathbb{P}^{\xi, t}}\left[\left(M_{T}^{\eta, v^{T}}\right)^{q}\right]^{1-p}=\frac{x^{p}}{p}\left(v^{T}(t, y)\right)^{\delta} \mathbb{E}^{\mathbb{P}^{\xi, t}}\left[D_{t, T}^{v^{T}}\right]^{1-p} .
$$


Here $\xi=(x, y)$ and $\left(\mathbb{P}^{\xi, t}\right)_{\xi}$ is the solution to the martingale problem for $L$ in the canonical state space whose coordinate process starts from time $t$. Therefore, thanks to duality results for power utility between payoffs and stochastic discount factors (Guasoni and Robertson, 2009, Lemma 5), the claims will follow if $D^{v^{T}}$ is a $\mathbb{P}^{y}$ martingale for all $y \in E$. It suffices to show $1=\mathbb{E}^{\mathbb{P}^{y}}\left[D_{T}^{v^{T}}\right]$. It follows from (4.4) that

$$
\frac{v_{y}^{T}}{v^{T}}=\frac{\hat{v}_{y}}{\hat{v}}+\frac{h_{y}^{T}}{h^{T}}
$$

Using this, the $\mathbb{P}^{y}$ independence of $Y$ and $B$ implies (Karatzas and Kardaras, 2007, Lemma 4.8)

$$
\begin{aligned}
& \mathbb{E}^{\mathbb{P}^{y}}\left[D_{T}^{v^{T}}\right] \\
& =\mathbb{E}^{\mathbb{P}^{y}}\left[\mathcal{E}\left(\int\left(-q \Upsilon^{\prime} \Sigma^{-1} \mu+A \frac{v_{y}^{T}}{v^{T}}\right)^{\prime} \frac{1}{a} d W-q \int\left(\Sigma^{-1} \mu+\Sigma^{-1} \Upsilon \delta \frac{v_{y}^{T}}{v^{T}}\right)^{\prime} \sigma \bar{\rho} d B\right)_{T}\right], \\
& =\mathbb{E}^{\mathbb{P}^{y}}\left[\mathcal{E}\left(\int\left(-q \Upsilon^{\prime} \Sigma^{-1} \mu+A\left(\frac{\hat{v}_{y}}{\hat{v}}+\frac{h_{y}^{T}}{h^{T}}\right)\right)^{\prime} \frac{1}{a} d W-q \int\left(\Sigma^{-1} \mu+\Sigma^{-1} \Upsilon \delta\left(\frac{\hat{v}_{y}}{\hat{v}}+\frac{h_{y}^{T}}{h^{T}}\right)\right)^{\prime} \sigma \bar{\rho} d B\right)_{T}\right], \\
& =\mathbb{E}^{\mathbb{P}^{y}}\left[\mathcal{E}\left(\int\left(-q \Upsilon^{\prime} \Sigma^{-1} \mu+A\left(\frac{\hat{v}_{y}}{\hat{v}}+\frac{h_{y}^{T}}{h^{T}}\right)\right)^{\prime} \frac{1}{a} d W\right)_{T}\right], \\
& =\mathbb{E}^{\mathbb{P}^{y}}\left[\mathcal{E}\left(\int\left(-q \Upsilon^{\prime} \Sigma^{-1} \mu+A\left(\frac{\hat{v}_{y}}{\hat{v}}+\frac{h_{y}^{T}}{h^{T}}\right)\right)^{\prime} \frac{1}{a} d W-q \int\left(\Sigma^{-1} \mu+\Sigma^{-1} \Upsilon \delta \frac{\hat{v}_{y}}{\hat{v}}\right)^{\prime} \sigma \bar{\rho} d B\right)_{T}\right] .
\end{aligned}
$$

Note that for $w=\hat{v}$ the process of (4.11) specifies to

$$
D_{t}^{\hat{v}}=\mathcal{E}\left(\int\left(-q \Upsilon^{\prime} \Sigma^{-1} \mu+A \frac{\hat{v}_{y}}{\hat{v}}\right)^{\prime} \frac{1}{a} d W-q \int\left(\Sigma^{-1} \mu+\Sigma^{-1} \Upsilon \delta \frac{\hat{v}_{y}}{\hat{v}}\right)^{\prime} \sigma \bar{\rho} d B\right)_{t} .
$$

This is precisely the stochastic exponential that changes the dynamics from $\mathbb{P}^{y}$ to those for $\hat{\mathbb{P}}^{y}$. It follows from part (ii) of Lemma 4.3 and the backward martingale theorem (Cheridito et al., 2005, Remark 2.3.2) that $D^{\hat{v}}$ is a $\left(\mathbb{P}^{y},\left(\mathcal{F}_{t}\right)_{t \geq 0}\right)$ martingale, whence

$$
\left.\frac{d \hat{\mathbb{P}}^{y}}{d \mathbb{P}^{y}}\right|_{\mathcal{F}_{t}}=D_{t}^{\hat{v}}
$$

Furthermore, the Brownian motion $\hat{W}$ from (2.18) is related to $W$ by $d \hat{W}_{t}=d W_{t}+\left(q \rho \sigma^{\prime} \Sigma^{-1} \mu-\right.$ $\left.a \hat{v}_{y} / \hat{v}\right) d t$. Using this, for all $t \leq T$

$$
\begin{gathered}
\mathcal{E}\left(\int\left(-q \Upsilon^{\prime} \Sigma^{-1} \mu+A\left(\frac{\hat{v}_{y}}{\hat{v}}+\frac{h_{y}^{T}}{h^{T}}\right)\right)^{\prime} \frac{1}{a} d W-q \int\left(\Sigma^{-1} \mu+\Sigma^{-1} \Upsilon \delta \frac{\hat{v}_{y}}{\hat{v}}\right)^{\prime} \sigma \bar{\rho} d B\right)_{t} \\
=D_{t}^{\hat{v} \mathcal{E}}\left(\int a \frac{h_{y}^{T}}{h^{T}} d \hat{W}\right)_{t}=D_{t}^{\hat{v}} \frac{h^{T}\left(t, Y_{t}\right)}{h^{T}(0, y)} .
\end{gathered}
$$


The last equality follows from the fact that $h^{T}$ solves the differential expression in (4.5) combined with Ito's formula. The second to last equality follows from the identity for any adapted, integrable processes $a, b$ and Wiener process $W$ that

$$
\mathcal{E}\left(\int\left(a_{s}+b_{s}\right) d W_{s}\right)=\mathcal{E}\left(\int a_{s} d W_{s}\right) \mathcal{E}\left(\int b_{s} d W_{s}-\int b_{s} a_{s} d s\right) .
$$

Using (4.27) and (4.26) in (4.24) and applying Proposition 4.5 it holds that

$$
\mathbb{E}^{\mathbb{P}^{y}}\left[D_{T}^{v^{T}}\right]=\mathbb{E}^{\hat{\mathbb{P}}^{y}}\left[\frac{h^{T}\left(T, Y_{T}\right)}{h^{T}(0, y)}\right]=1,
$$

which is the desired result.

4.3. Conditional densities and wealth processes. The last prerequisite for the main result is to relate the terminal wealths resulting from using the finite horizon optimal strategies $\pi^{T}$ of (2.22) and the long-run optimal strategy $\hat{\pi}$ of (2.23). Recall the definition of $D^{w}$ from (4.11), and consider $w=v^{T}$ and $w=\hat{v}$. A similar calculation to (4.27) using (4.23) and (4.25) gives

$$
D_{t}^{v^{T}}=D_{t}^{\hat{v}} \frac{h^{T}\left(t, Y_{t}\right)}{h^{T}(0, y)} \mathcal{E}\left(-\int a \frac{h_{y}^{T}}{h^{T}} \Delta^{\prime} d \hat{B}\right)_{t}
$$

where

$$
\Delta=q \delta \rho^{\prime} \bar{\rho}
$$

and the Brownian Motion $\hat{B}$ is from (2.18) and related to $B$ by $\hat{B}=B+q \bar{\rho} \sigma^{\prime} \Sigma^{-1} \mu+\Delta^{\prime} a \hat{v}_{y} / \hat{v}$. Dividing by $D_{t}^{\hat{v}}$ gives

$$
\frac{D_{t}^{v^{T}}}{D_{t}^{\hat{v}}}=\frac{h^{T}\left(t, Y_{t}\right)}{h^{T}(0, y)} \mathcal{E}\left(-\int a \frac{h_{y}^{T}}{h^{T}} \Delta^{\prime} d \hat{B}\right)_{t} .
$$

For $w=v^{T}$ and $\lambda=0$, (4.14) gives (since all assumptions hold) almost surely $\mathbb{P}^{y}$ (and hence $\hat{\mathbb{P}}^{y}$ ) for any $0 \leq t \leq T$

$$
\left(X_{t}^{0, T}\right)^{p}=\left(X_{t}^{\pi, v^{T}}\right)^{p}=x^{p} D_{t}^{v^{T}}\left(\frac{v^{T}(0, y)}{v^{T}\left(t, Y_{t}\right)}\right)^{\delta}=x^{p} D_{t}^{v^{T}} e^{\delta \lambda_{c} t}\left(\frac{\hat{v}(y) h^{T}(0, y)}{\hat{v}\left(Y_{t}\right) h^{T}\left(t, Y_{t}\right)}\right)^{\delta},
$$

where the last equality uses (4.4). Similarly, for $w=\hat{v}$ and $\lambda=\lambda_{c}$, it follows that for the long-run optimal strategy $\hat{\pi}$ defined in (2.23), (4.14) gives the $\mathbb{P}^{y}\left(\hat{\mathbb{P}}^{y}\right)$ almost sure equality, for each $0 \leq t \leq T$

$$
\left(\hat{X}_{t}\right)^{p}=\left(X_{t}^{\hat{\pi}}\right)^{p}=x^{p} D_{t}^{\hat{v}} e^{\delta \lambda_{c} t}\left(\frac{\hat{v}(y)}{\hat{v}\left(Y_{t}\right)}\right)^{\delta} .
$$

Therefore, (4.30), (4.31) and (4.29) imply

$$
\frac{X_{t}^{0, T}}{\hat{X}_{t}}=\left(\frac{D_{t}^{v^{T}}}{D_{t}^{\hat{v}}}\right)^{1 / p}\left(\frac{h^{T}\left(t, Y_{t}\right)}{h^{T}(0, y)}\right)^{-\delta / p}=\left(\frac{h^{T}\left(t, Y_{t}\right)}{h^{T}(0, y)}\right)^{\frac{1-\delta}{p}} \mathcal{E}\left(-\int a \Delta \frac{h_{y}^{T}}{h^{T}} d \hat{B}\right)_{t}^{\frac{1}{p}} .
$$

where the last equality uses (4.29). Equations (4.29) and (4.32) will be used in the next section. 
Remark 4.8. The proof of Proposition 4.7 showed $D^{v^{T}}$ is a $\left(\mathbb{P}^{y},\left(\mathcal{F}_{t}\right)_{0 \leq t \leq T}\right)$ martingale for each $y \in E$. Thus, (4.22) and (4.30) in conjunction with (2.4) implies that

$$
D_{t}^{v^{T}}=\left.\frac{d \mathbb{P}^{T, y}}{d \mathbb{P}^{y}}\right|_{\mathcal{F}_{t}} .
$$

\subsection{Proof of main results in Section 2.2.}

Proof of Lemma 2.11. By (Pinsky, 1995, Theorem 5.1.5), Assumptions 2.8 and 2.9 ensure a solution, $\left(\mathbb{P}_{Y}^{y}\right)_{y \in E}$ to the martingale problem for $\widetilde{\mathcal{L}}$ on $E$, where

$$
\widetilde{\mathcal{L}}=\frac{1}{2} A \partial_{y y}^{2}+b \partial_{y} .
$$

Let $\xi=(z, y) \in \mathbb{R}^{d} \times E$. The result now follows by considering the family of measures $\left(\mathbb{P}^{\xi}:=\right.$ $\left.W^{z, d} \times \mathbb{P}_{Y}^{y}\right)_{\xi \in \mathbb{R}^{d} \times E}$ on $(\Omega, \mathcal{F})$ where $W^{z, d}$ is $d$-dimensional Wiener measure corresponding to a Brownian Motion starting at $z$.

Proof of Proposition 2.16. By Theorem 18 in Guasoni and Robertson (2009), under Assumptions 2.8, 2.9, and 2.13, (2.20) yields the existence of a function $\hat{v}$ which satisfies (2.12), (2.14), along with the first inequality in (2.15). By Holder's inequality, (2.19) ensures that the second inequality in (2.15) holds as well, proving the assertion.

Proof of Lemma 2.18. Recall the notation of Section 4.3, From (4.26), (4.33) and (4.29), the limit in (2.24) holds provided that 6 :

$$
\hat{\mathbb{P}}^{y}-\lim _{T \rightarrow \infty} \frac{h^{T}\left(t, Y_{t}\right)}{h^{T}(0, y)} \mathcal{E}\left(-\int a \Delta \frac{h_{y}^{T}}{h^{T}} d \hat{B}\right)_{t}=1 .
$$

where $\Delta$ is from (4.28). Set $L_{t}^{T}=h^{T}\left(t, Y_{t}\right) / h^{T}(0, y)$. Proposition 4.5implies that a) for each $T, L^{T}$ is a positive $\hat{\mathbb{P}}^{y}$ martingale on $[0, T]$ with expectation 1 and b) for each $t \geq 0, \lim _{T \rightarrow \infty} L_{t}^{T}=1$ almost surely $\hat{\mathbb{P}}^{y}$. Therefore, Fatou's lemma gives $1 \geq \lim _{T \rightarrow \infty} \mathbb{E}^{\hat{\mathbb{P}}^{y}}\left[L_{t}^{T}\right] \geq \mathbb{E}^{\hat{\mathbb{P}}^{y}}\left[\liminf _{T \rightarrow \infty} L_{t}^{T}\right]=$ 1 , which implies $\lim _{T \rightarrow \infty} \mathbb{E}^{\hat{\mathbb{P}}^{y}}\left[\left|L_{t}^{T}-1\right|\right]=0$ by Scheffé's lemma. As shown in (4.27), $L_{t}^{T}=$ $\mathcal{E}\left(\int a h_{y}^{T} / h^{T} d \hat{W}\right)_{t}$. Lemma 3.9 thus yields

$$
\hat{\mathbb{P}}^{y}-\lim _{T \rightarrow \infty}\left[\int a \frac{h_{y}^{T}}{h^{T}} d \hat{W}, \int a \frac{h_{y}^{T}}{h^{T}} d \hat{W}\right]_{t}=0 .
$$

Observing that $\|\Delta\|^{2}$ is a constant (by Assumption 2.13), the previous identity implies that $\hat{\mathbb{P}}^{y}$ $\lim _{T \rightarrow \infty}\left[\int a \Delta h_{y}^{T} / h^{T} d \hat{B}, \int a \Delta h_{y}^{T} / h^{T} d \hat{B}\right]_{t}=0$, whence $\hat{\mathbb{P}}^{y} \lim _{T \rightarrow \infty} \int_{0}^{t} a \Delta h_{y}^{T} / h^{T} d \hat{B}=0$, which implies $\hat{\mathbb{P}}^{y}-\lim _{T \rightarrow \infty} \mathcal{E}\left(\int a \Delta h_{y}^{T} / h^{T} d \hat{B}\right)_{t}=1$, i.e., the second term on the left-hand-side of (4.34) also converges to 1 . This concludes the proof of (4.34).

\footnotetext{
${ }^{6}$ The notation $\mathbb{P}^{y}$ - lim is short for the limit in probability
} 
Proof of Theorem 2.19. Let $\mathcal{S}_{T}$ be either $\left\{\sup _{u \in[0, t]}\left|r_{u}^{T}-1\right| \geq \epsilon\right\}$ or $\left\{\left[\Pi^{T}, \Pi^{T}\right]_{t} \geq \epsilon\right\}$, which are both $\mathcal{F}_{t}$-measurable. It follows from Theorem 2.5 and Remark 2.6 part iii) that

$$
\lim _{T \rightarrow \infty} \mathbb{E}^{\hat{\mathbb{P}}^{y}}\left[\left.\frac{d \mathbb{P}^{T, y}}{d \hat{\mathbb{P}}^{y}}\right|_{\mathcal{F}_{t}} 1_{\mathcal{S}_{T}}\right]=0 .
$$

On the other hand, (2.24) and Scheffé's lemma combined imply that

$$
\lim _{T \rightarrow \infty} \mathbb{E}^{\hat{\mathbb{P}}^{y}}\left[\left|\frac{d \mathbb{P}^{T, y}}{d \hat{\mathbb{P}}^{y}}\right|_{\mathcal{F}_{t}}-1 \mid\right]=0 .
$$

Hence, combining the previous identity with (4.35), it follows that $\lim _{T \rightarrow \infty} \hat{\mathbb{P}}^{y}\left(\mathcal{S}_{T}\right)=0$. Since $\hat{\mathbb{P}}^{y} \sim \mathbb{P}$ on $\mathcal{F}_{t}$ from Proposition 4.6, it follows that

$$
\lim _{T \rightarrow \infty} \mathbb{P}^{y}\left(\mathcal{S}_{T}\right)=\lim _{T \rightarrow \infty} \mathbb{E}^{\hat{\mathbb{P}}^{y}}\left[\left.\frac{d \mathbb{P}^{y}}{d \hat{\mathbb{P}}^{y}}\right|_{\mathcal{F}_{t}} 1_{\mathcal{S}_{T}}\right]=0,
$$

where the last equality follows from the dominated convergence theorem.

Proof of Theorem 2.20. A similar argument to the one in the proof of Lemma 2.18, combined with (4.32), yields that $\hat{\mathbb{P}}^{y}-\lim _{T \rightarrow \infty} X_{t}^{0, T} / \hat{X}_{t}=1$. On the other hand, Theorem 2.19 part a), combined with the equivalence between $\mathbb{P}$ and $\hat{\mathbb{P}}^{y}$, implies that $\hat{\mathbb{P}}^{y}-\lim _{T \rightarrow \infty} X_{t}^{1, T} / X_{t}^{0, T}=1$. Hence the last two identities combined give $\hat{\mathbb{P}}^{y}-\lim _{T \rightarrow \infty} \hat{r}_{t}^{T}=1$. Now recall that $\hat{\pi}$ is the optimal portfolio for the logarithmic investor under $\hat{\mathbb{P}}^{y}$, it then follows from the numéraire property of $\hat{\pi}$ that $\hat{r}^{T}$ is a $\hat{\mathbb{P}}^{y}$ supermartingale, which implies that $\lim _{T \rightarrow \infty} \mathbb{E}^{\hat{\mathbb{P}}^{y}}\left[\left|\hat{r}_{t}^{T}-1\right|\right]=0$, by Fatou's lemma and Scheffé's lemma. As a result, the statements follow applying Lemma 3.9 under the probability $\hat{\mathbb{P}}^{y}$, and remain valid under the equivalent probability $\mathbb{P}^{y}$.

\section{REFERENCES}

S. Benninga and J. Mayshar. Heterogeneity and option pricing. Review of Derivatives Research, 4 (1):7-27, 2000.

T.R. Bielecki and S.R. Pliska. Risk sensitive asset management with transaction costs. Finance Stoch., 4(1):1-33, 2000. ISSN 0949-2984.

T.R. Bielecki, D. Hernandez-Hernandez, and S.R. Pliska. Risk sensitive asset management with constrained trading strategies. In Recent developments in mathematical finance (Shanghai, 2001), pages 127-138. World Sci. Publishing, River Edge, NJ, 2002.

A. Buraschi, P. Porchia, and F. Trojani. Correlation risk and optimal portfolio choice. Journal of Finance, 65(1):393-420, 2010.

P. Cheridito, D. Filipović, and M. Yor. Equivalent and absolutely continuous measure changes for jump-diffusion processes. The Annals of Applied Probability, 15(3):1713-1732, 2005.

J.C. Cox and C. Huang. A continuous-time portfolio turnpike theorem. Journal of Economic Dynamics and Control, 16(3-4):491-507, 1992. 
J. Cvitanic and S. Malamud. Asset Prices, Funds' Size and Portfolio Weights in Equilibrium with Heterogeneous and Long-Lived Funds, 2008. Working paper, ETH Zurich and Caltech.

F. Delbaen and W. Schachermayer. A general version of the fundamental theorem of asset pricing. Mathematische Annalen, 300(3):463-520, 1994.

F. Delbaen and W. Schachermayer. The fundamental theorem of asset pricing for unbounded stochastic processes. Mathematische Annalen, 312(2), 1998.

J. Detemple and M. Rindisbacher. Dynamic asset allocation: Portfolio decomposition formula and applications. Review of Financial Studies, 23(1):25-100, 2010.

P.H. Dybvig, L.C.G. Rogers, and K. Back. Portfolio turnpikes. Review of Financial Studies, 12 (1):165-195, 1999.

L.C. Evans. Partial differential equations, volume 19 of Graduate Studies in Mathematics. American Mathematical Society, Providence, RI, 1998. ISBN 0-8218-0772-2.

W.H. Fleming and W.M. McEneaney. Risk-sensitive control on an infinite time horizon. SIAM J. Control Optim., 33(6):1881-1915, 1995. ISSN 0363-0129.

W.H. Fleming and S.J. Sheu. Risk-sensitive control and an optimal investment model. Math. Finance, 10(2):197-213, 2000. ISSN 0960-1627. INFORMS Applied Probability Conference (Ulm, 1999).

W.H. Fleming and S.J. Sheu. Risk-sensitive control and an optimal investment model. II. Ann. Appl. Probab., 12(2):730-767, 2002. ISSN 1050-5164.

A. Friedman. Partial differential equations of parabolic type. Prentice-Hall Inc., Englewood Cliffs, N.J., 1964.

A. Friedman. Stochastic differential equations and applications. Vol. 1. Academic Press [Harcourt Brace Jovanovich Publishers], New York, 1975. Probability and Mathematical Statistics, Vol. 28.

P. Guasoni and S. Robertson. Portfolios and risk permia for the long run. To appear in Ann. Appl. Probab., 2009.

N.H. Hakansson. Convergence to isoelastic utility and policy in multiperiod portfolio choice. Journal of Financial Economics, 1(3):201-224, 1974.

D. Heath and M. Schweizer. Martingales versus PDEs in finance: an equivalence result with examples. Journal of Applied Probability, 37:947-957, 2000.

C-F. Huang and T. Zariphopoulou. Turnpike behavior of long-term investments. Finance Stoch., 3(1):15-34, 1999.

G. Huberman and S. Ross. Portfolio turnpike theorems, risk aversion, and regularly varying utility functions. Econometrica, 51(5):1345-1361, 1983.

X. Jin. Consumption and portfolio turnpike theorems in a continuous-time finance model. $J$. Econom. Dynam. Control, 22(7):1001-1026, 1998. 
J. Kallsen. Optimal portfolios for exponential Lévy processes. Mathematical Methods of Operations Research, 51(3):357-374, 2000.

I. Karatzas and C. Kardaras. The numéaire portfolio in semimartingale financial models. Finance Es Stochastics, 11:447-493, 2007.

I. Karatzas and G. Žitković. Optimal consumption from investment and random endowment in incomplete semimartingale markets. The Annals of Probability, 31(4), 2003.

C. Kardaras. The continuous behavior of the numéraire portfolio under small changes in information structure, probabilistic views and investment constraints. Stochastic Processes and their applications, 120(3):331-347, 2010.

D. Kramkov and M. Sîrbu. On the two-times differentiability of the value functions in the problem of optimal investment in incomplete markets. The Annals of Applied Probability, 16(3):13521384, 2006a.

D. Kramkov and M. Sîrbu. Sensitivity analysis of utility-based prices and risk-tolerance wealth processes. The Annals of Applied Probability, 16(4):2140-2194, 2006b.

D. Kramkov and M. Sîrbu. Asymptotic analysis of utility-based hedging strategies for small number of contingent claims. Stochastic Processes and their Applications, 117(11):1606-1620, 2007.

H. Leland. On turnpike portfolios. In K. Shell G. Szego, editor, Mathematical methods in investment and finance. North-Holland, Amsterdam, 1972.

J. Mossin. Optimal multiperiod portfolio policies. Journal of Business, 41(2):215-229, 1968.

H. Nagai and S. Peng. Risk-sensitive optimal investment problems with partial information on infinite time horizon. In Recent developments in mathematical finance (Shanghai, 2001), pages 85-98. World Sci. Publishing, River Edge, NJ, 2002a.

H. Nagai and S. Peng. Risk-sensitive dynamic portfolio optimization with partial information on infinite time horizon. Ann. Appl. Probab., 12(1):173-195, 2002b. ISSN 1050-5164.

Y. Pinchover. Large time behavior of the heat kernel and the behavior of the Green function near criticality for nonsymmetric elliptic operators. J. Funct. Anal., 104(1):54-70, 1992. ISSN 0022-1236.

R.G. Pinsky. Positive harmonic functions and diffusion, volume 45 of Cambridge Studies in Advanced Mathematics. Cambridge University Press, Cambridge, 1995.

L.C.G. Rogers and D. Williams. Diffusions, Markov processes, and martingales. Vol. 2. Cambridge Mathematical Library. Cambridge University Press, Cambridge, 2000. ISBN 0-521-77593-0. Itô calculus, Reprint of the second (1994) edition.

S. Ross. Portfolio turnpike theorems for constant policies. Journal of Financial Economics, 1(2): 171-198, 1974.

T. Zariphopoulou. A solution approach to valuation with unhedgable risks. Finance 83 Stochastics, $5(1): 61-82,2001$. 
(Paolo Guasoni) School of Mathematical Sciences, Dublin City University, Glasnevin, Dublin 9, Ireland. Department of Mathematics and Statistics, Boston University, 111 Cummington st, Boston, MA 02215, USA

E-mail address: guasoni@bu.edu

(Constantinos Kardaras) Department of Mathematics and Statistics, Boston University, 111 CummingTON st, Boston, MA 02215, USA

E-mail address: kardaras@bu.edu

(Scott Robertson) Department of Mathematical Sciences, Wean Hall 6113, Carnegie Mellon UniverSity, PitTsburgh, PA 15213, USA

E-mail address: scottrob@andrew.cmu.edu

(Hao Xing) Department of Statistics, London School of Economics and Political Science, 10 Houghton ST, LONDON, WC2A $2 \mathrm{AE}, \mathrm{UK}$

E-mail address: h.xing@lse.ac.uk 